Al1106 978900

NBSIR 83-1684

\title{
TENSILE, COMPRESSIVE, AND SHEAR PROPERTIES OF A 64-kg/m 3 POLYURETHANE FOAM AT LOW TEMPERATURES
}

\author{
J.M. Arvidson
}

L.L. Sparks

Chen Guobang

National Bureau of Standards

U.S. Department of Commerce

Boulder, Colorado 80303

February 1983 

NBSIR 83-1684

\title{
TENSILE, COMPRESSIVE, AND SHEAR \\ PROPERTIES OF A $64 \cdot \mathrm{kg} / \mathrm{m}^{3}$ \\ POLYURETHANE FOAM AT \\ LOW TEMPERATURES
}

\section{J.M. Arvidson* \\ L.L. Sparks* * \\ Chen Guobang†}

\author{
${ }^{\star}$ Fracture and Deformation Division \\ National Measurement Laboratory \\ * Thermophysical Properties Division \\ National Engineering Laboratory \\ National Bureau of Standards \\ U.S. Department of Commerce \\ Boulder, Colorado 80303
}

†Guest worker at NBS. Department of Thermal Science, Zhejiang University, Hangzhon, Zhejiang Province, People's Republic of China

February 1983

Prepared for:

Gas Research Institute

8600 West Bryn Mawr Avenue

Chicago, Illinois 60631

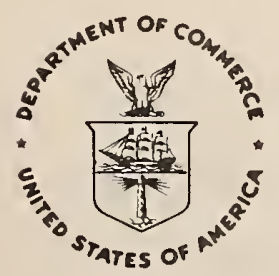

U.S. DEPARTMENT OF COMMERCE, Malcolm Baldrige, Secretary

NATIONAL BUREAU OF STANDARDS, Ernest Ambler, Director 

Tensile, Compressive, and Shear Properties of a $64-\mathrm{kg} / \mathrm{m}^{3}$

Polyurethane Foam at Low Temperatures

J. M. Arvidson, L. L. Sparks, and Chen Guobang

Polyurethane foam, having a density of $64 \mathrm{~kg} / \mathrm{m}^{3}$, was tested at 295, 111 , 76, and $4 \mathrm{~K}$. The material properties reported are Young's moduTus, proportional limit, yield strength (at $0.2 \%$ offset), tensile, shear, and compressive strengths, and elongation (elastic and plastic). To perform these tests, a unique apparatus was developed. This apparatus permits tension, compression, and shear testing of materials at any temperature ranging from 295 to $1.8 \mathrm{~K}$. Strain is measured with a concentric, overlapping-cylinder capacitance extensometer that is highly sensitive and linear in output.

Key words: compressive strength; elongation; foam; insulation; low temperatures; mechanical properties; proportional 1imit; shear strength; tensile strength; yield strength; Young's modulus. 



\section{INTRODUCTION}

Polyurethane foams are used in many structural as well as insulating applications in which knowledge of material performance in extreme environments (e.g., at liquefied natural gas, liquid nitrogen, and liquid helium temperatures) is essential. Accurate data, predictive capability, and standardized methods and materials improve the selection and development of materials for these applications. The mechanical properties reported here are part of a broader effort to understand and predict the thermal and mechanical behavior of expanded plastics (foams) in cryogenic environments.

The tensile, compressive, and shear properties of polyurethane foam, having a density of $64 \mathrm{~kg} / \mathrm{m}^{3}$, were determined using a test fixture developed specifically for this program [1]. This fixture provides the capability of determining the above material properties at any temperature from ambient $(295 \mathrm{~K})$ to $1.8 \mathrm{~K}$. Tests can be done in a cold gaseous atmosphere or in liquids, such as helium $(4 \mathrm{~K})$, nitrogen $(76 \mathrm{~K})$, and dry ice and alcohol (195 K). The fixture has also been designed to test materials at the above temperatures while under static pressures ranging from subatmospheric to approximately $0.3 \mathrm{MPa}(43.7 \mathrm{psia})$.

A strain extensometer that was developed for use with soft viscoelastic materials utilizes concentric overlapping cylinders and the change in electrical capacitance to detect specimen strain [2]. This type of extensometer does not attach directly to the specimen, so the effect of instrumentation on the experimental results is negligible (verified to contribute $<2.5 \times 10^{-8} \mathrm{~m}$ for our specimen geometry). The extensometer works well in cryogenic environments, is accurate, and is linear for large strains $[3,4]$. The capacitance extensometer system designed for this work had a linearity range in excess of $2.5 \mathrm{~cm}$ when used with the current foam specimen geometry (2.9-cm diameter by $9.9-\mathrm{cm}$ length). 
Similar extensometers can be designed for specific sensitivities and extents of linearity [5]. As long as the capacitance extensometer is situated in a stable fluid (i.e., single phase and nonboiling) the resultant is a very high signal-to-noise ratio. These sensors function in gases as well as in liquids and the device is only sensitive to the dielectric constant of the test medium. The original calibration of the system, for example, can be performed at room temperature in air. To conduct a test in any other medium (e.g., liquid nitrogen) the original calibration need only be corrected for the change in dielectric constant [6].

\section{MATERIAL CHARACTERIZATION}

The material tested in this study is a nominal $64-\mathrm{kg} / \mathrm{m}^{3}$ polyurethane foam designated as GM35. This amorphous organic polymer is a thermosetting foam. Our supply of this material was obtained from the NBS Office of Standard Reference Materials (OSRM) in Gaithersburg, Maryland. The OSRM distributed this and other expanded plastics for the Products Research Committee $[7,8]$. These materials were commercially produced and designated as General Materials.

The bulk supply of GM35 was a $0.1-\mathrm{m} \times 0.6-\mathrm{m} \times 0.6-\mathrm{m}$ slab (figure $1 \mathrm{a}$ ). The orientation of the elongated cell axis for the material used in the physical properties tests was determined optically from statistical evaluation of photomicrographs. The ratios of cell height to cell width for the principal orthogonal planes of the physical test specimens were: $x / y=0.94 \pm$ $0.34, z / x=1.60 \pm 0.65, z / y=1.51 \pm 0.58$. The uncertainties given represent estimates of one standard deviation. The cell orientations relative to the orthogonal axes of the bulk slab are shown in figure $1 b$. 
The density of this resin-gas composite was found to be $62.22 \pm 0.28$ $\mathrm{kg} / \mathrm{m}^{3}, 62.52 \pm 0.11 \mathrm{~kg} / \mathrm{m}^{3}$, and $62.56 \pm 0.13 \mathrm{~kg} / \mathrm{m}^{3}$ after conditioning at $23^{\circ} \mathrm{C}$ and 24,50 , and 92 percent relative humidity respectively. The uncertainties given are estimates of one standard deviation.

\section{SPECIMENS}

Test specimens taken from the $x / y$ or $z / x$ plane in the bulk supply (see figure 1) are designated "longitudinal" when loaded parallel to the $z$ direction. Conversely, specimens taken from the $x / y$ plane and loaded parallel to either the $x$ or $y$ direction are designated "transverse".

The tensile specimens were rods $9.9-\mathrm{cm}$ long and $2.9-\mathrm{cm}$ in diameter. This specimen was used to determine Young's modulus, proportional 1imit, yield strength (at $0.2 \%$ offset), and elongation (extrapolated using ultimate tensile strength). For the determination of ultimate tensile strength, a reduced section specimen was used. Its gage length was $5.1 \mathrm{~cm}$ and its diameter was $1.9 \mathrm{~cm}$. All tensile specimens were epoxied to polycarbonate grips, which were threaded to accomodate the tensile pull-rod system. The reduced-section geometry forced fracture to occur within the gage length, thereby eliminating the effect of biaxial stresses at the grip ends.

The compression specimens were $2.54-\mathrm{cm}$ long and $2.9-\mathrm{cm}$ in diameter. These specimens did not require grips.

Shear specimens were $1.9 \mathrm{~cm} \times 2.54 \mathrm{~cm} \times 0.4 \mathrm{~cm}$. The specimens were epoxied to two flat plates and each plate was attached to the upper and lower tensile pull-rod system, respectively. An aluminum cylinder was slipped over the specimen and plates to help maintain alignment and minimize induced torque during testing. In addition, the cylinder has a built-in resistive heater that is controlled by a thermocouple to produce and maintain the desired test temperature. 


\section{TEST PROCEDURES}

Most tests were conducted at $295 \mathrm{~K}$ (air), $111 \mathrm{~K}$ (gaseous helium), $76 \mathrm{~K}$ (1 iquid nitrogen), and $4 \mathrm{~K}$ (liquid helium). To minimize thermal shock to the specimen, the liquid helium or nitrogen was transferred at a very slow rate. The tensile and compressive properties included: Young's modulus, proportional limit, yield strength (at 0.2 percent offset), tensile and compressive strengths, and elongation (elastic and plastic).

Since an ASTM shear strength test method is not available for soft cellular materials such as these foams, several methods were carefully considered, and a version of the guillotine-type shear test was selected. Many specimen geometries (thicknesses and widths) were tested to find the best combination that most consistently failed in shear. At least three tests were conducted at each temperature; in some cases, more tests were run to determine material variability. Prior to testing, all specimens were "conditioned" in an environmental chamber for not less than four days at $23^{\circ} \mathrm{C}$ and 50 percent relative humidity. Each specimen was tested shortly after removal from the environmental chamber. All tests were conducted in a conventional compression/ tension test machine at a strain rate of $5 \times 10^{-3} \mathrm{~min}^{-1}$. (Varying the rate from $5 \times 10^{-4}$ to $5 \times 10^{-2} \mathrm{~min}^{-1}$ had no measurable effect on results.)

\section{RESULTS}

Results are presented in figures 2 through 15 and tables 1 through 6 . The bars on the figures indicate the data spread from replicate tests. Scatter is typically higher for compression and shear than for tensile tests; this is because the compressive and shear tests are more sensitive to problems such as misalignment (tensile specimens self-align during the test). Unlike the $32-\mathrm{kg} / \mathrm{m}^{3}$ foam that was tested previously [1], the cell orientation of the $64-\mathrm{kg} / \mathrm{m}^{3}$ material agreed very well with the bun-line orientation 
(i.e., the vertical or "rise" direction is denoted as longitudinal). Tensile results shown in figures 2 through 6 are Young's modulus, ultimate strength, and strain-versus-temperature, as well as stress-versus-strain curves for all temperatures. In addition to the above properties, compressive results include yield strength (at $0.2 \%$ offset), proportional limit versus temperature, and a typical load-versus-displacement curve, as shown in figures 7 through 14. The shear results are given in figure 15 .

Tensile, Young's modulus, proportional limit, yield strength, ultimate strength, and shear strength values all increase as temperature decreases from $295 \mathrm{~K}$ to $4 \mathrm{~K}$. The longitudinal orientation results have the highest values. As shown in figure 4, tensile strain decreases with temperature, and the material becomes brittle at temperatures below $111 \mathrm{~K}$. The compressive Young's modulus versus temperature, as shown in figure 7 , indicates a decrease in mean values from $111 \mathrm{~K}$ to $76 \mathrm{~K}$, for both longitudinal and transverse specimens. The same behavior was also noted for a $32-\mathrm{kg} / \mathrm{m}^{3}$ polyurethane foam material that was previously tested [1]. Using the Student's "t" test for small sample size, the longitudinal and transverse compressive data were calculated for 76 and $111 \mathrm{~K}$. The results indicate a level of confidence of $>99.95 \%$ for the transverse and $88 \%$ for longitudinal tests that the mean compressive modulus at $76 \mathrm{~K}$ is lower than the mean compressive modulus at $111 \mathrm{~K}$ [9]. Figure 11 indicates a deformation capability during compression at low temperatures. However, since the polyurethane material is incapable of true plastic deformation at cryogenic temperatures, this apparent plastic strain is caused by individual cells that collapse during testing. Figures 12 and 13 show the stress-versus-strain behavior of longitudinal and transverse specimens at 295 , 111, 76, and $4 \mathrm{~K}$. Figure 14 is an actual record of load-versus-displacement for a typical specimen. The load drops illustrate points 
at which individual or multiple cells fail under stress. Owing to material variability, the stress-strain graphs were plotted as smooth curves, which do not show the cell failure phenomena. Each stress-strain curve, at a given temperature and orientation, is the average of three or more specimens tested.

\section{DISCUSSION}

Polyurethane foam behavior is typical of polymers in general, with large mechanical property changes such as a complete loss of ductility and a doubling of $E_{T}$ (Young's modulus-tension) following a temperature reduction from $295 \mathrm{~K}$ to $4 \mathrm{~K}$. Certain longitudinal properties, like shear strength and $\mathrm{E}_{\mathrm{C}}$ (Young's modulus-compression), exhibit maximum values between the two extreme temperature limits; however this is probably not a true material behavior but an effect influenced by material variability and orientation irregularities.

The anomalous behavior of compressive Young's modulus as shown in figure 7 appears to be a real effect. As stated previously, the $32-\mathrm{kg} / \mathrm{m}^{3}$ polyurethane foam tested exhibited the same drop in modulus from 111 to $76 \mathrm{~K}$. In addition, a third density of polyurethane foam $\left(96-\mathrm{kg} / \mathrm{m}^{3}\right)$ has recently been tested and it shows the same trend.

Sectioning after testing revealed that cell orientation varied slightly from the orientation of the mold axes (see figure 1). It is generally thought that foam specimens from the center of a large billet have uniform cells oriented with respect to the vertical, but this is not true of foams formed on a continuous or bun-line production facility. The cell orientation reflects the movement of the foaming resin. However, as shown in this higher density foam, the problem of cell orientation with respect to vertical is not as severe as with less dense materials. Cells in the $x-z$ plane of the material reported here have only a 86 to $87^{\circ}$ inclination to the $x$-axis (figure 1 ). 
Specimens tested previously in liquid nitrogen [1] appeared to have slightly lower shear strength values than those tested at the same temperature in cold helium gas. This may be an indication of environmental sensitivity. Nitrogen at low temperatures is deleterious to a number of other polymeric materials. Thermoplastics have exhibited crazing or reduced fracture strengths, or both when tensile tested in cold liquid or gaseous nitrogen, but not in vacuum or helium environments [10]. Environmental effects must ultimately be taken into account in design applications.

The authors are indebted to Robert S. Bell and Ronald D. Kriz for their assistance in the preparation of this manuscript for publication.

\section{References}

[1] J. M. Arvidson and L. L. Sparks, "Low Temperature Mechanical Properties of a Polyurethane Foam", NBSIR 81-1654, National Bureau of Standards, Boulder, Colorado (November, 1981).

[2] R. P. Reed, J. M. Arvidson, and R. L. Durcholz, Tensile properties of polyurethane and polystyrene foams from 76 to $300 \mathrm{~K}$, in: "Advances in Cryogenic Engineering," Vol. 18, K. D. Timmerhaus, ed., Plenum Press, New York (1973), pp. 184-193.

[3] J. M. Roberts, R. B. Herring, and D. E. Hartman, The use of capacitance gauge sensors to make precision mechanical property measurements, in: "Materials Technology," American Society for Mechanical Engineers, New York (1968), pp. 87-96.

[4] "High-Temperature Capacitive Strain Measurement System," NASA Tech. Brief B75-10069, NASA (1975).

[5] P. C. F. Woldendale, Capacitive displacement transducers with high accuracy and resolution, J. Sci. Instrum. (J. Phys. E) 1:817 (1968).

[6] G. R. White, Measurement of thermal expansion at low temperatures, Cryogenics, 2:151 (1961).

[7] "Materials Bank Compendium of Fire Property Data," Products Research Committee, J. W. Lyons, chairman, National Bureau of Standards, Washington, D.C. (1980).

[8] W. G. Jurevic, "Structural Plastics Applications Handbook Supplement 1 Test Methods," Technical Report AFML-TR-67-332 (1969). 
[9] J. W. Dally, Statistical treatment of experimental data, Experimental Mechanics 19(11):421 (1979).

[10] A. Hiltner and E. Baer, Mechanical properties of polymers at cryogenic temperatures, Polymer 15:805 (1974). 
List of Figures

Figure 1(a). Bulk supply of bun-1 ine-produced, $64-\mathrm{kg} / \mathrm{m}^{3}$ polyurethane foam.

(b). Cell orientation relative to the major coordinate axes of the bulk supply.

Figure 2. Tensile Young's modulus versus temperature.

Figure 3. U1timate tensile strength versus temperature.

Figure 4. Tensile strain versus temperature.

Figure 5. Tensile stress versus strain (longitudinal orientation).

Figure 6. Tensile stress versus strain (transverse orientation).

Figure 7. Compressive Young's modulus versus temperature.

Figure 8. Compressive strength versus temperature.

Figure 9. Compressive proportional limit versus temperature.

Figure 10. Compressive yield strength versus temperature.

Figure 11. Compressive strain versus temperature.

Figure 12. Compressive stress versus strain (longitudinal orientation).

Figure 13. Compressive stress versus strain (transverse orientation).

Figure 14. Compressive load versus displacement (transverse orientation).

Figure 15. Shear strength versus temperature (longitudinal and transverse).

List of Tables

Table 1. Tensile test results for a $64-\mathrm{kg} / \mathrm{m}^{3}$ polyurethane foam.

Table 2. Compressive test results for a $64-\mathrm{kg} / \mathrm{m}^{3}$ polyurethane foam.

Table 3. Shear strength test results for a $64-\mathrm{kg} / \mathrm{m}^{3}$ polyurethane foam.

Table 4. Summary of tensile test results for a $64-\mathrm{kg} / \mathrm{m}^{3}$ polyurethane foam (average values.).

Table 5. Summary of compressive test results for a $64-\mathrm{kg} / \mathrm{m}^{3}$ polyurethane foam (average values).

Table 6. Summary of shear strength test results for a $64-\mathrm{kg} / \mathrm{m}^{3}$ polyurethane foam (average values). 


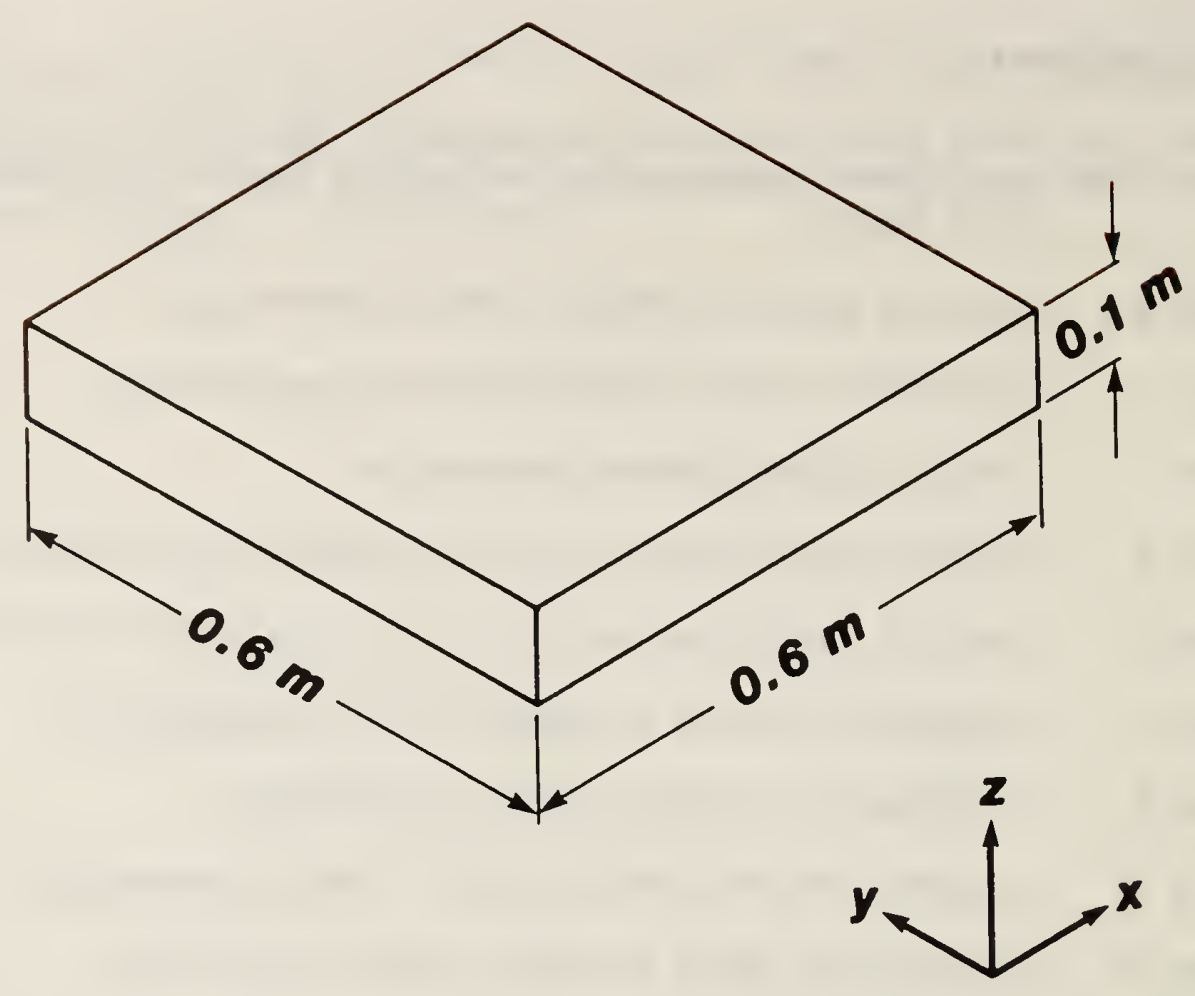

a)
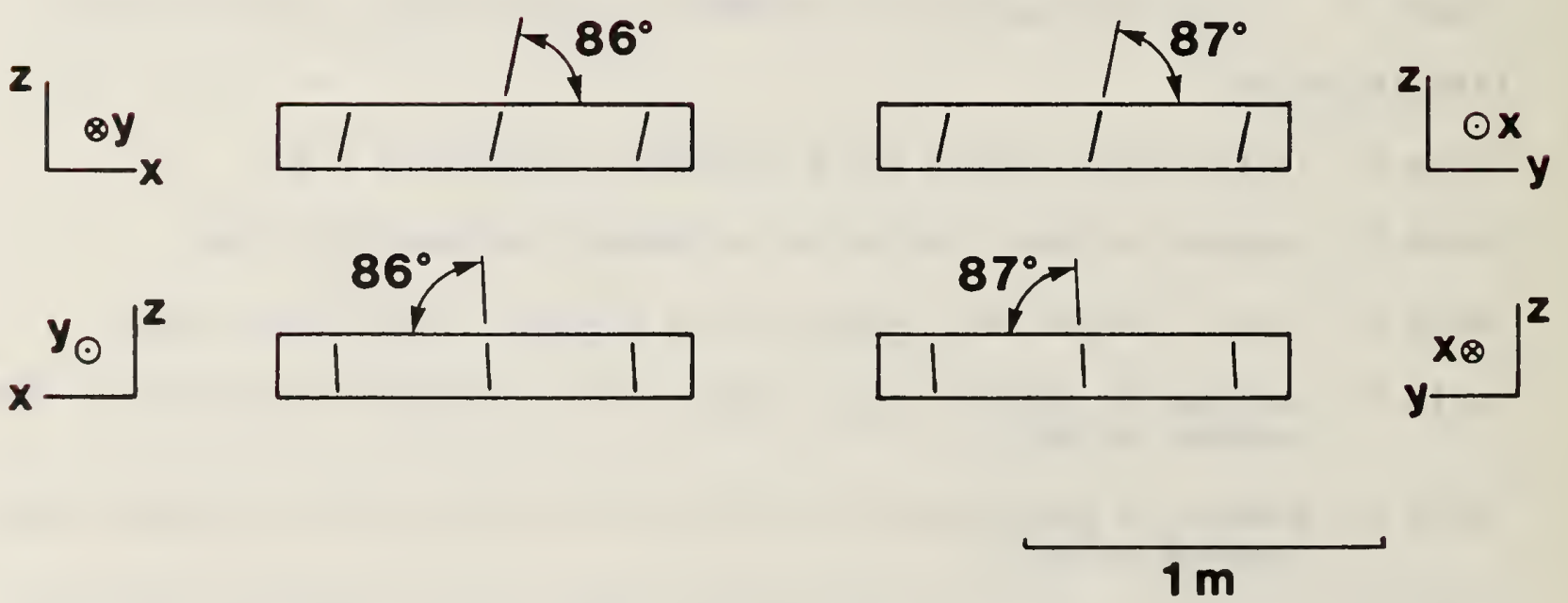

b)

Figure 1(a). Bulk supply of bun-1ine-produced, $64-\mathrm{kg} / \mathrm{m}^{3}$ polyurethane foam.

(b). Cell orientation relative to the major coordinate axes of the bulk supply. 


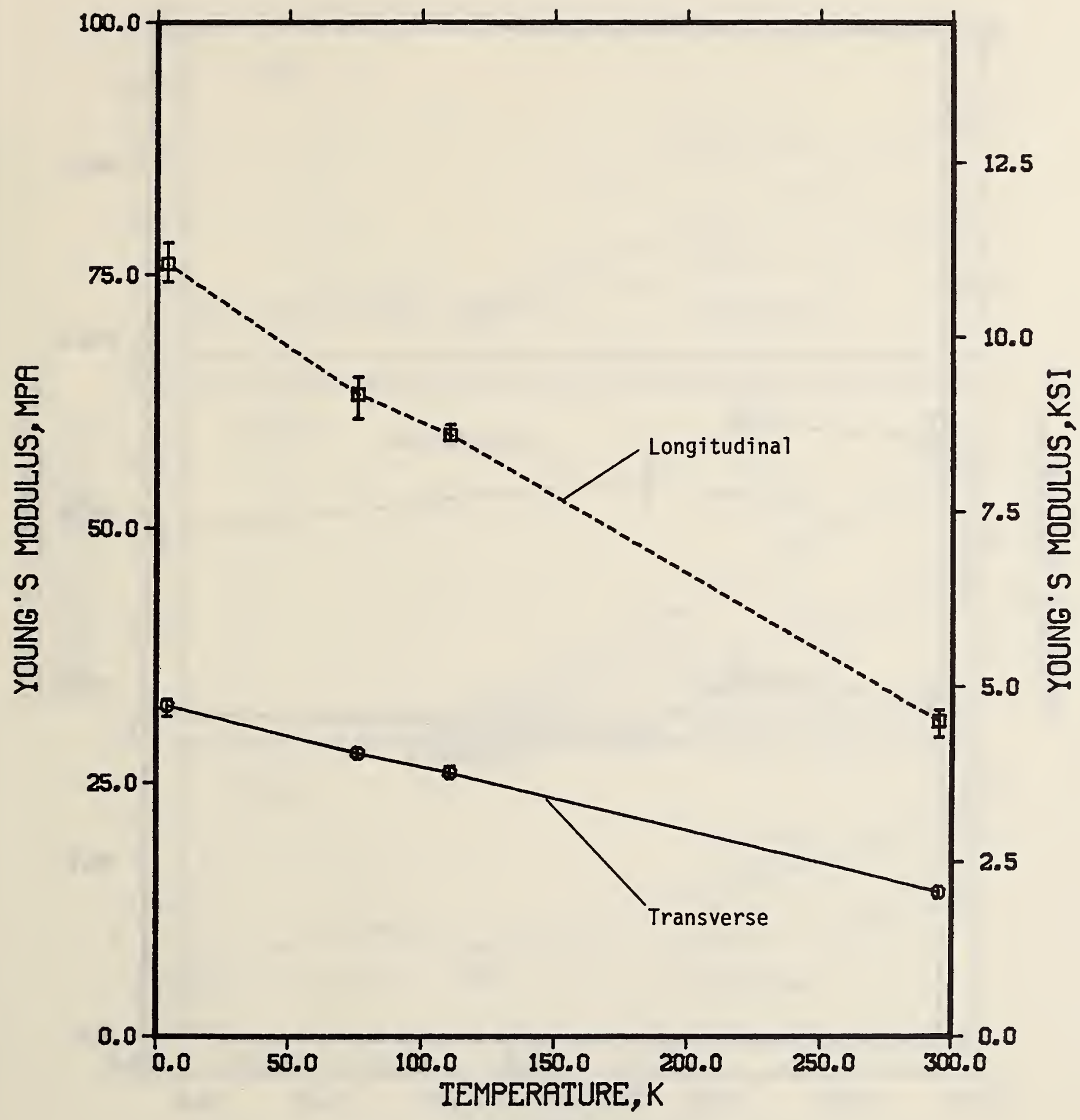

Figure 2. Tensile Young's modulus versus temperature. 


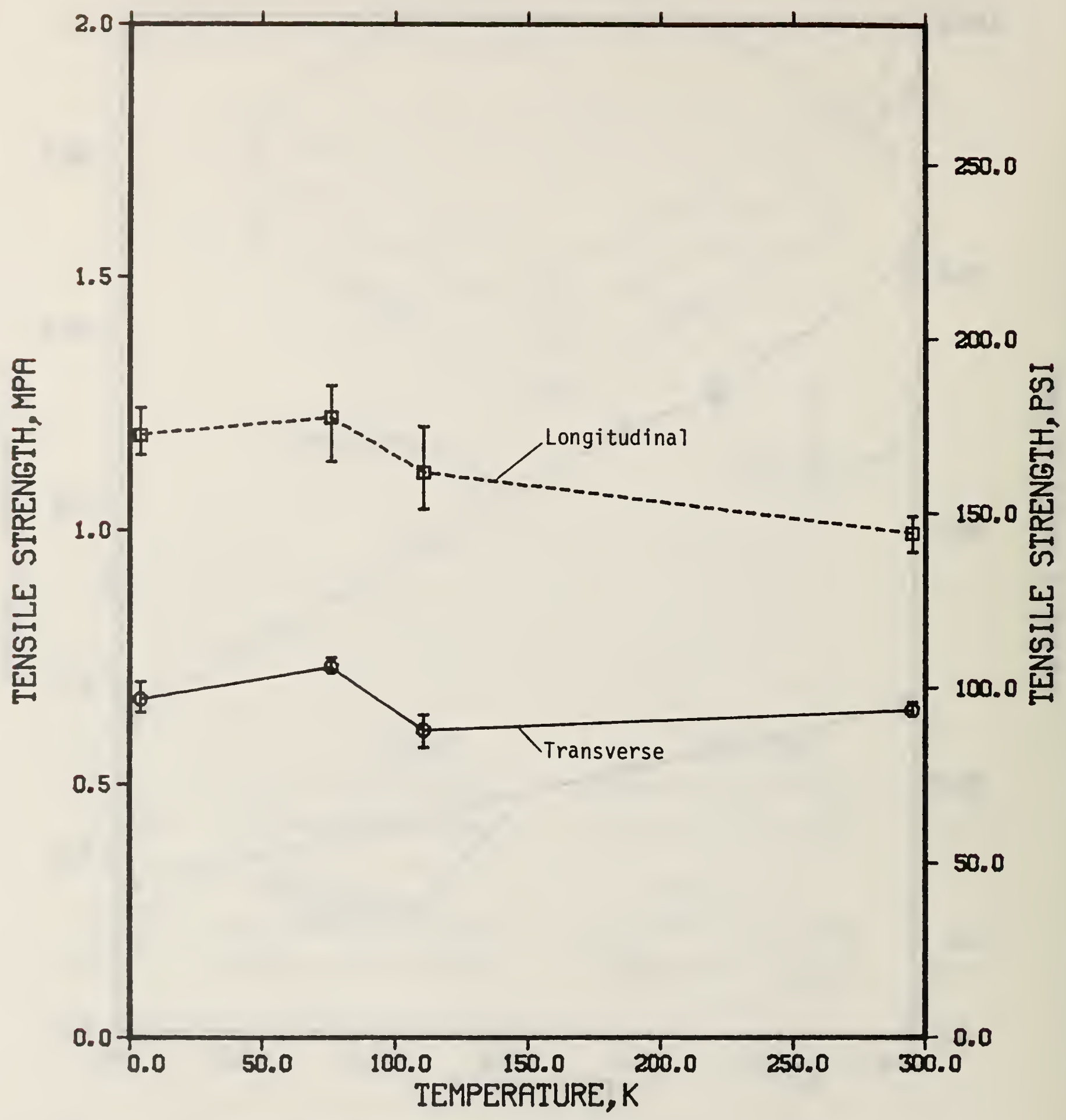

Figure 3. Ultimate tensile strength versus temperature. 


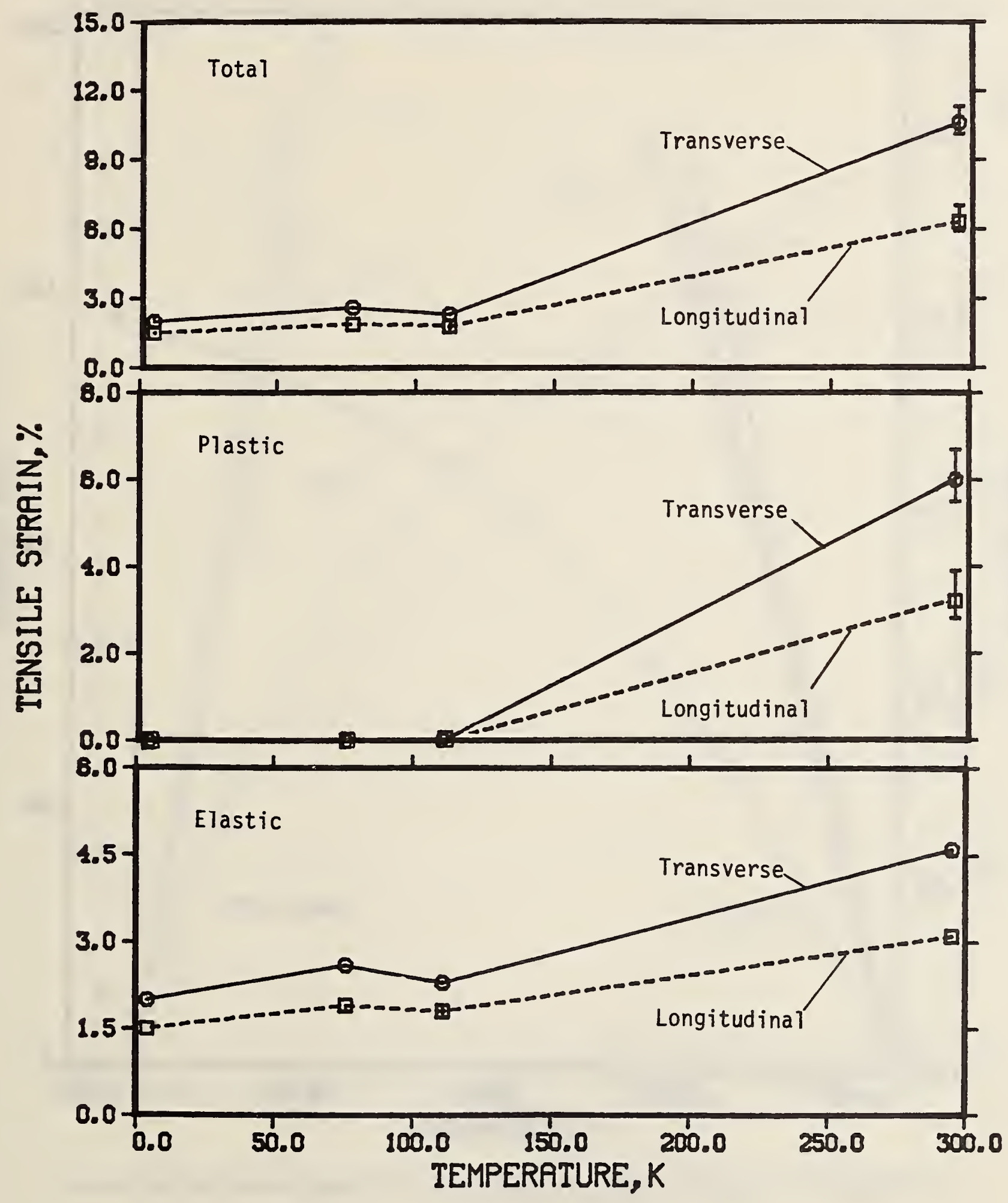

Figure 4. Tensile strain versus temperature. 


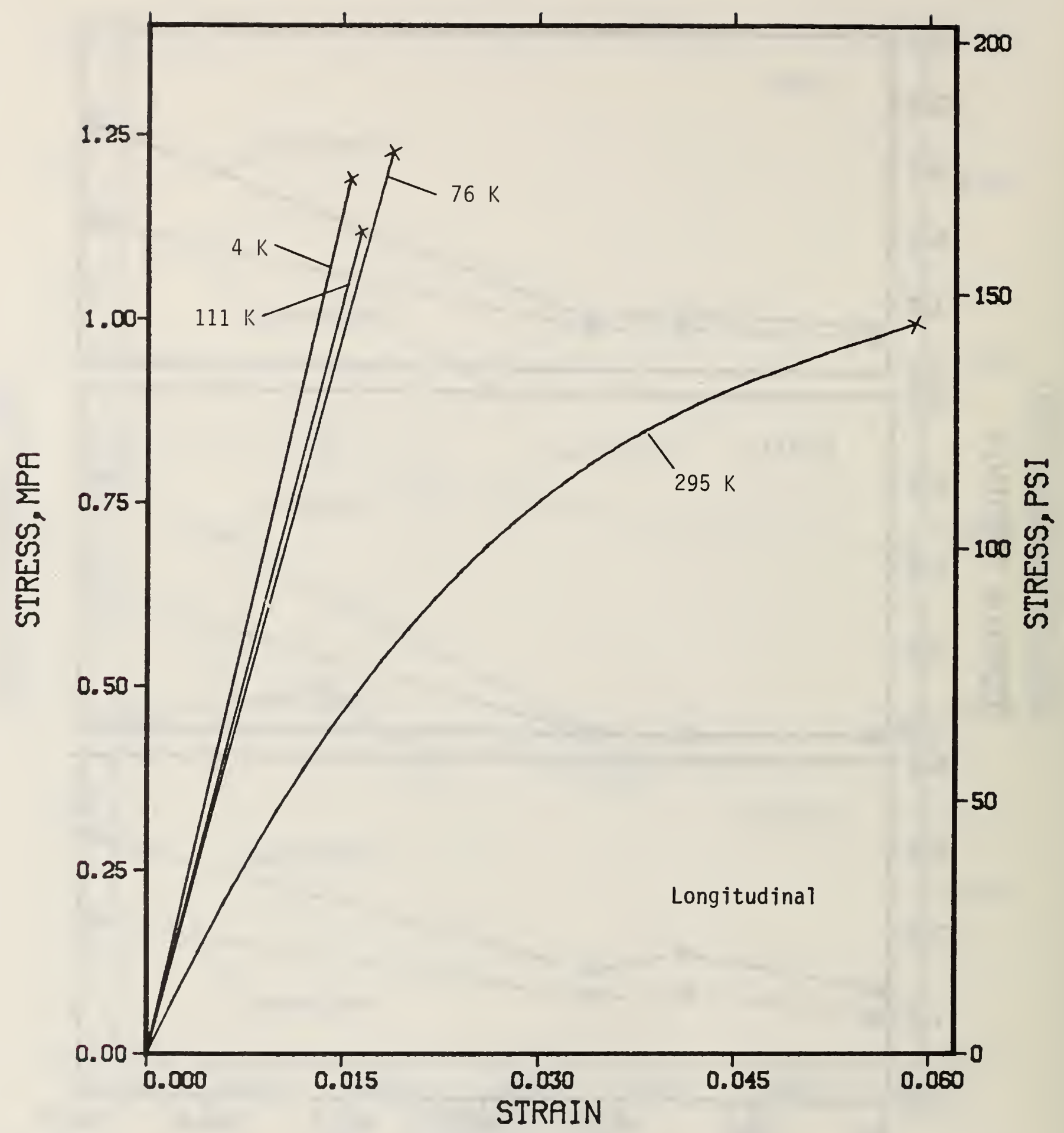

Figure 5. Tensile stress versus strain (longitudinal orientation). 


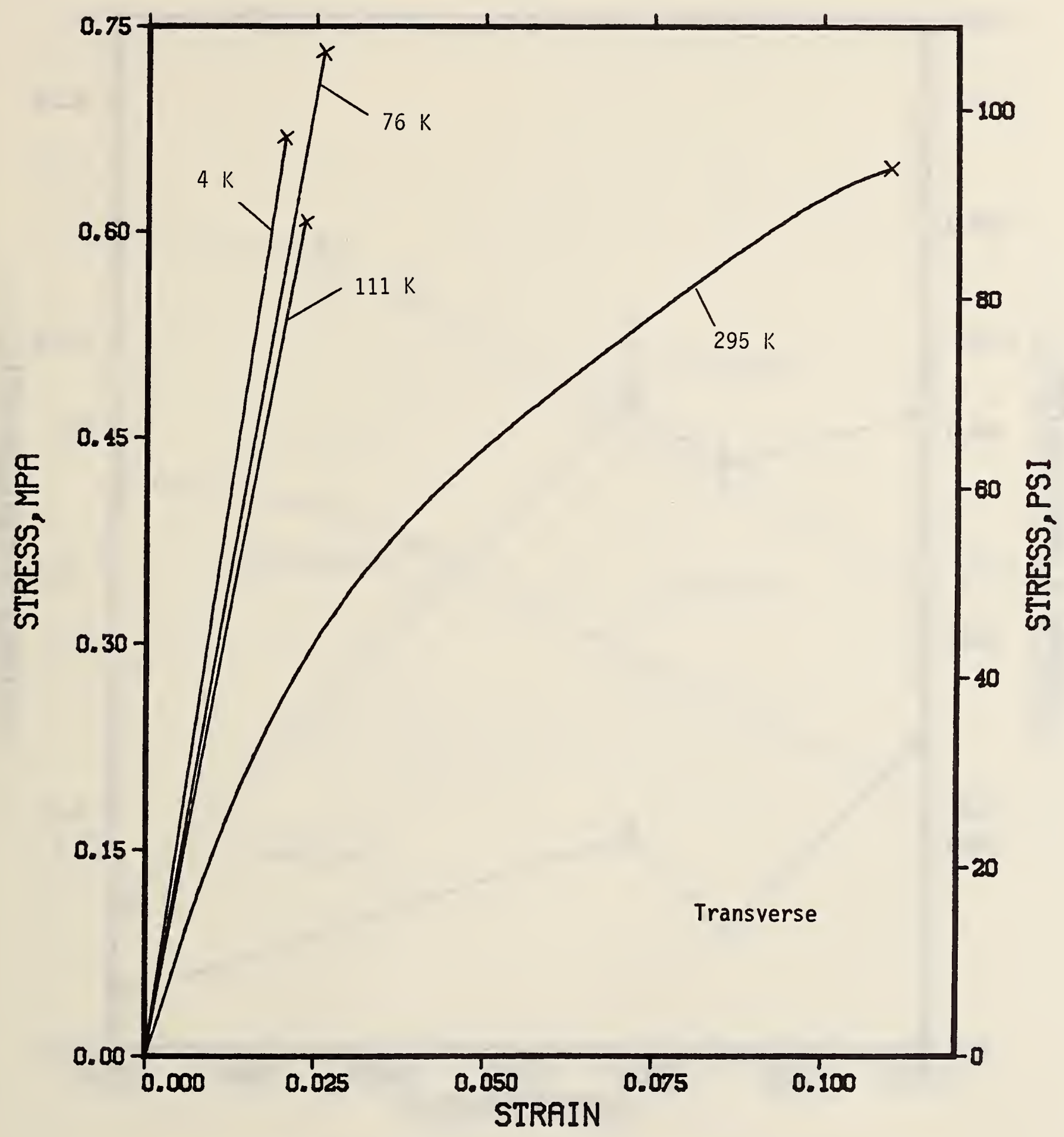

Figure 6. Tensile stress versus strain (transverse orientation). 


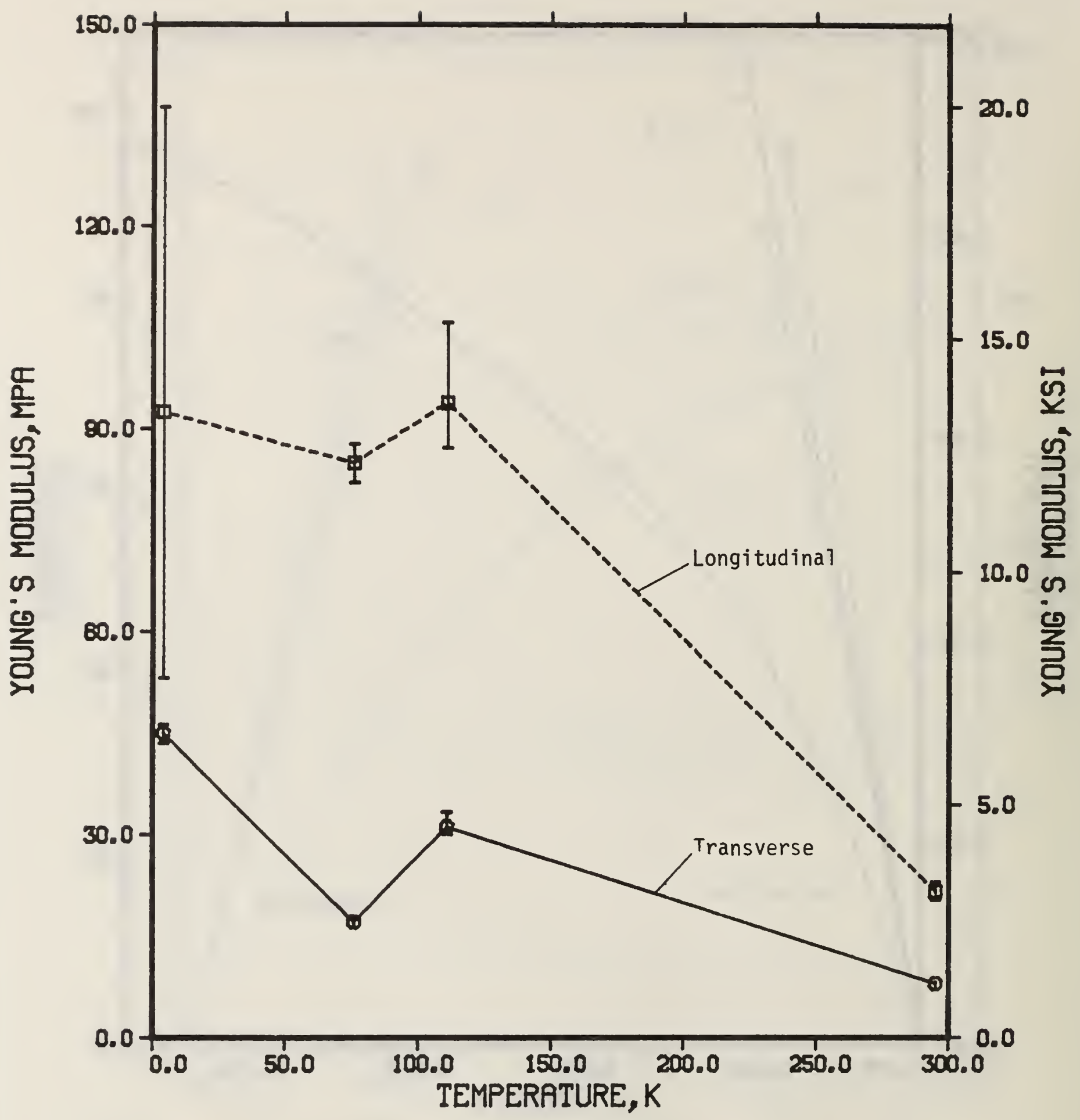

Figure 7. Compressive Young's modulus versus temperature. 


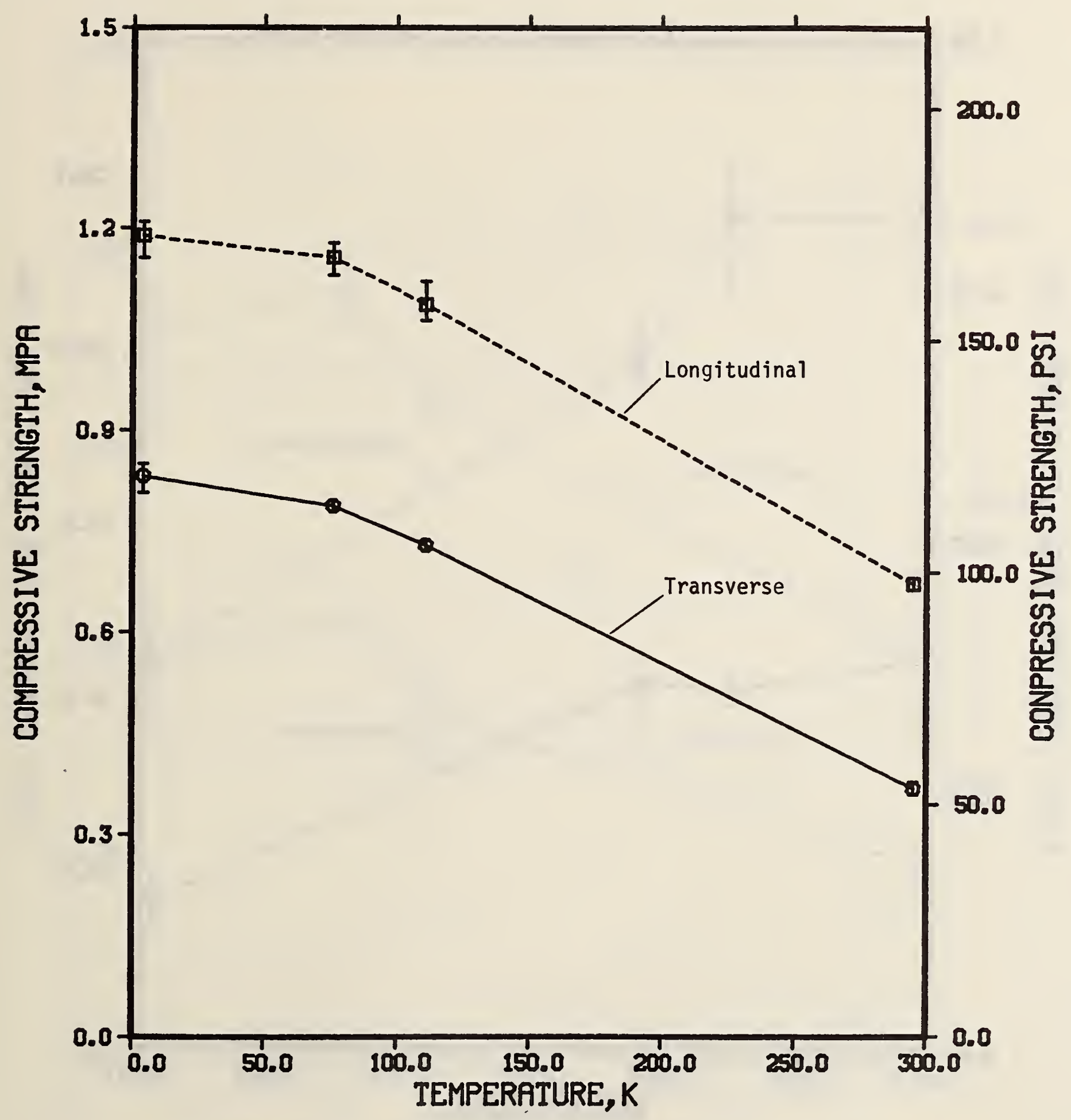

Figure 8. Compressive strength versus temperature. 


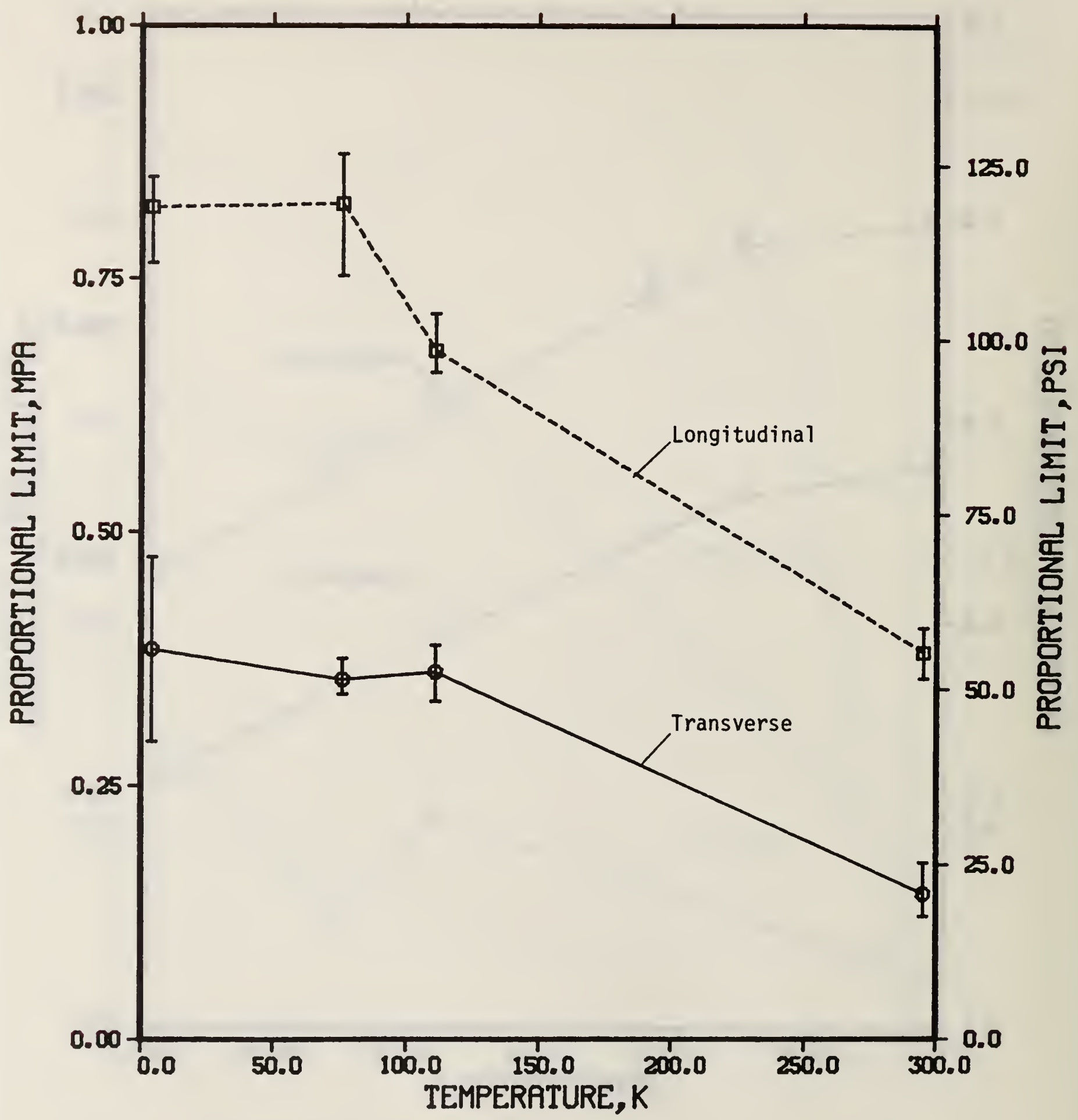

Figure 9. Compressive proportional limit versus temperature. 


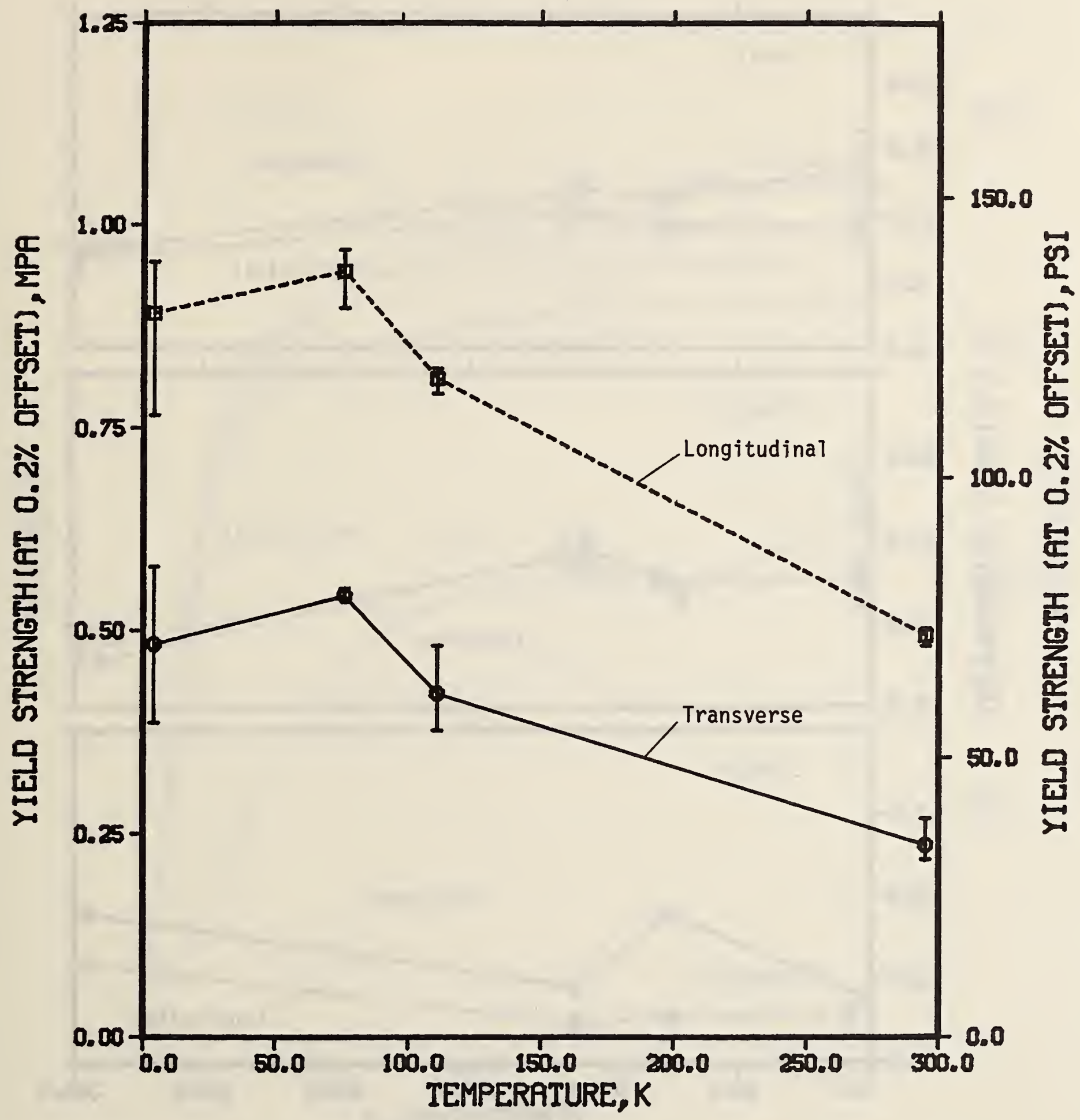

Figure 10. Compressive yield strength versus temperature. 


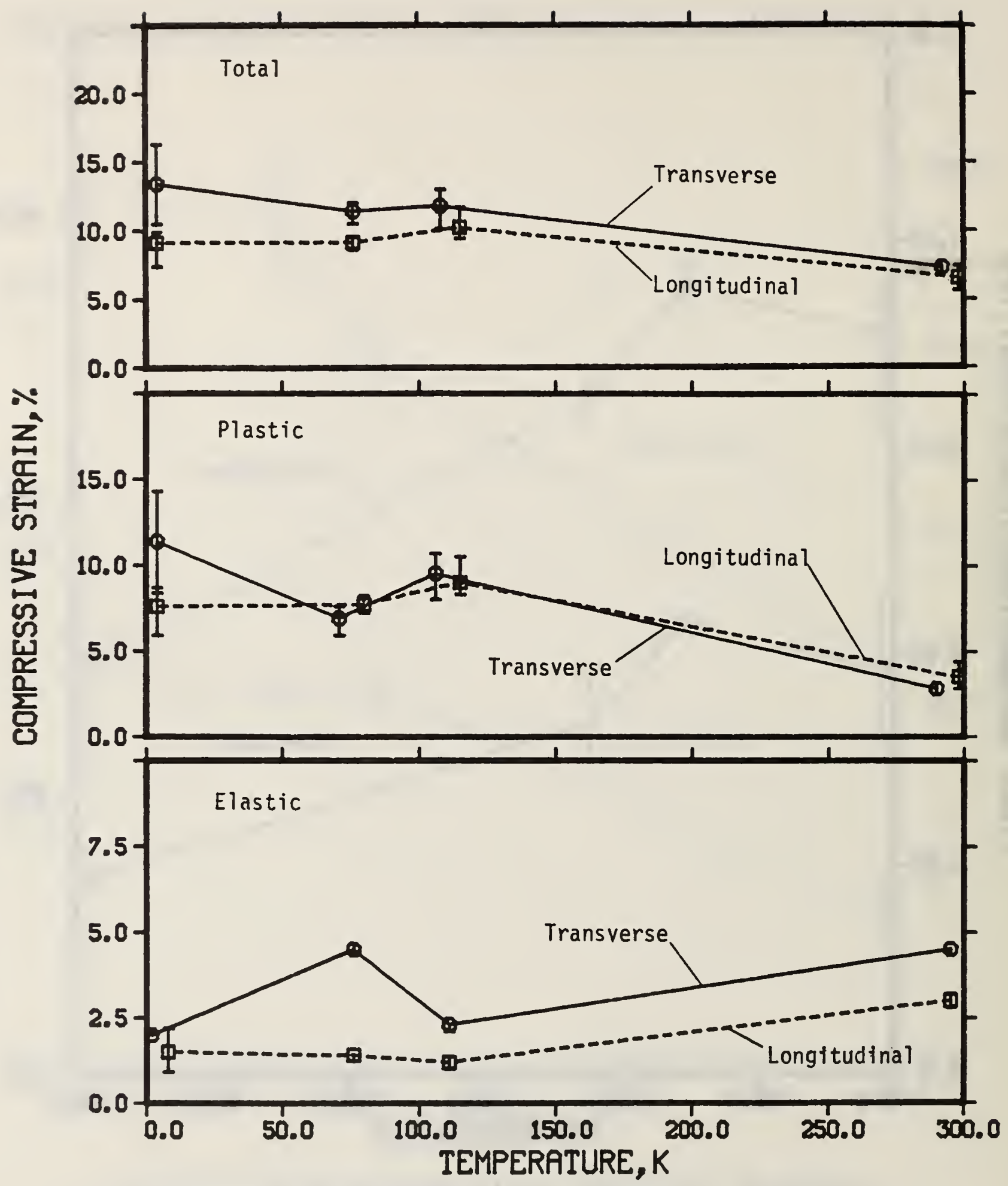

Figure 11. Compressive strain versus temperature. 


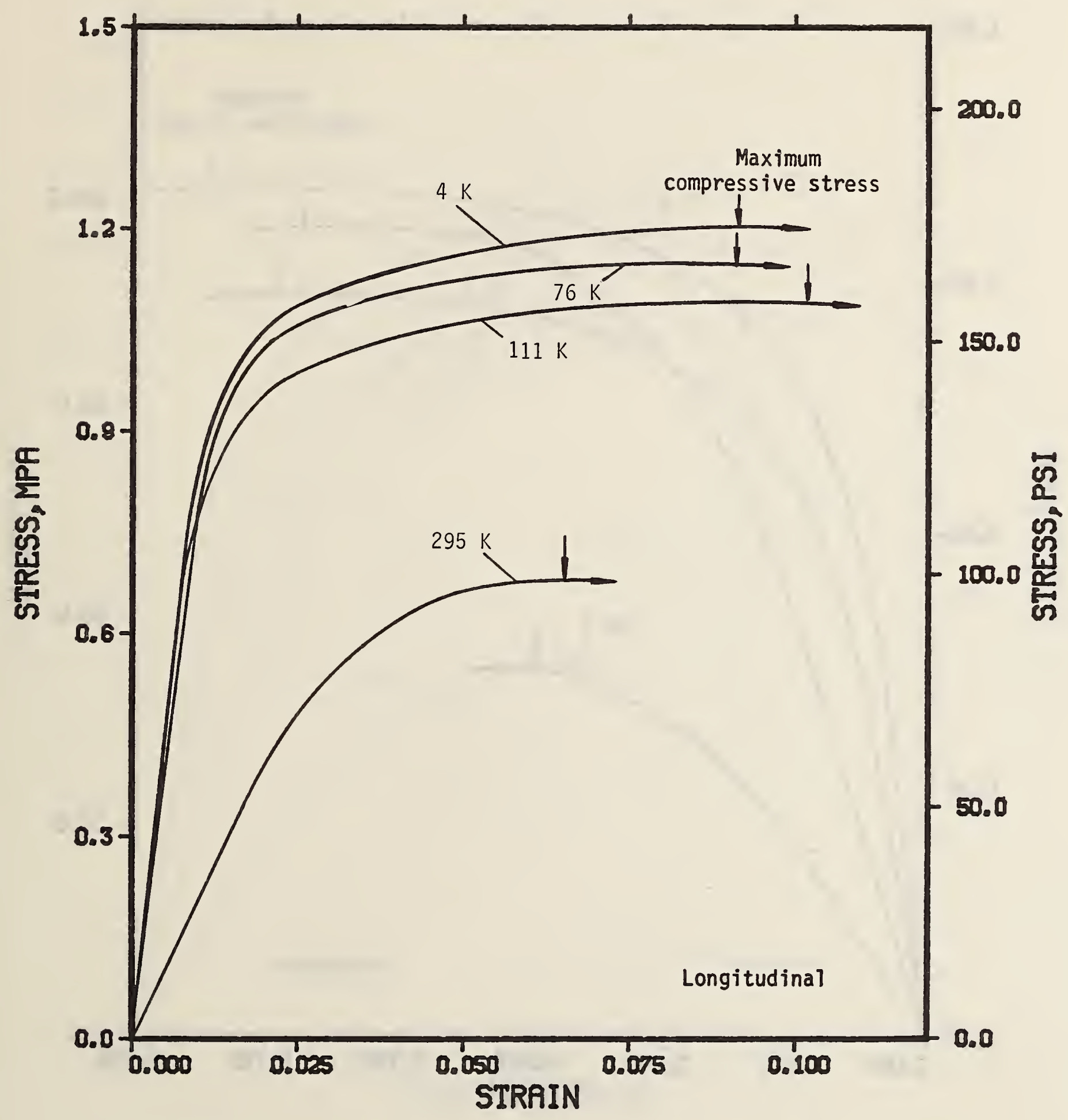

Figure 12. Compressive stress versus strain (longitudinal orientation). 


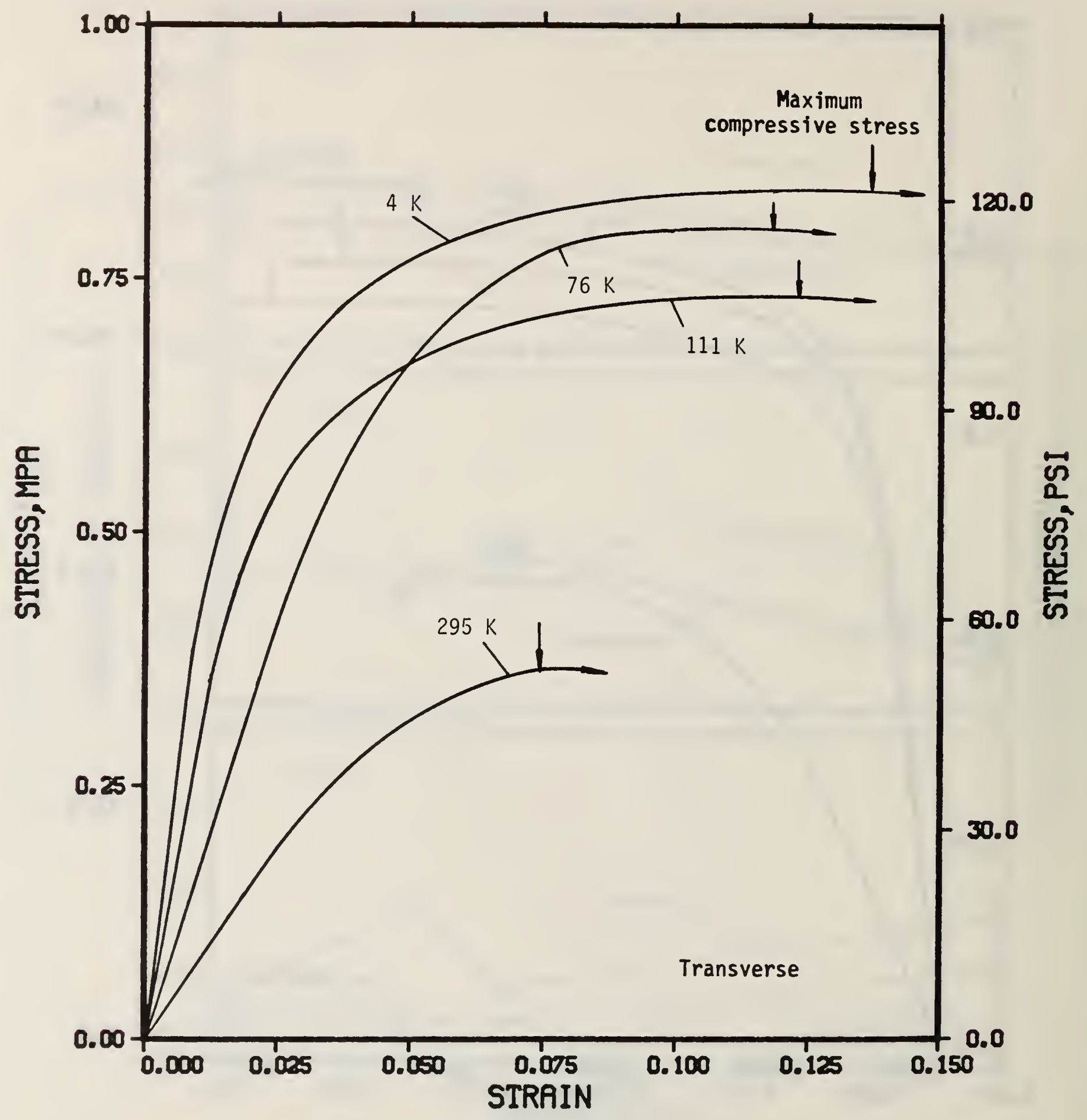

Figure 13. Compressive stress versus strain (transverse orientation). 


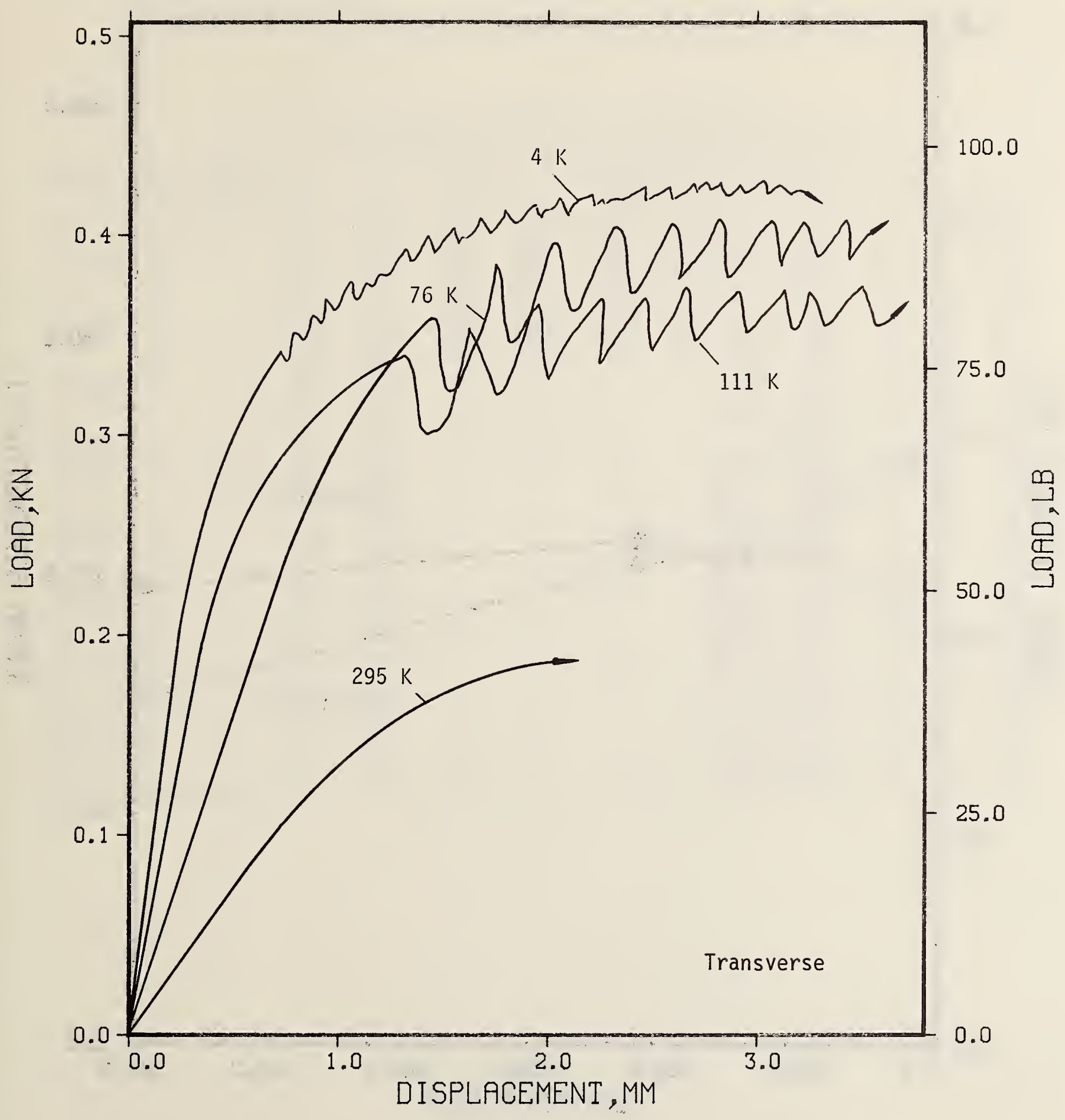

Figure 14. Compressive load versus displacement (transverse orientation). 


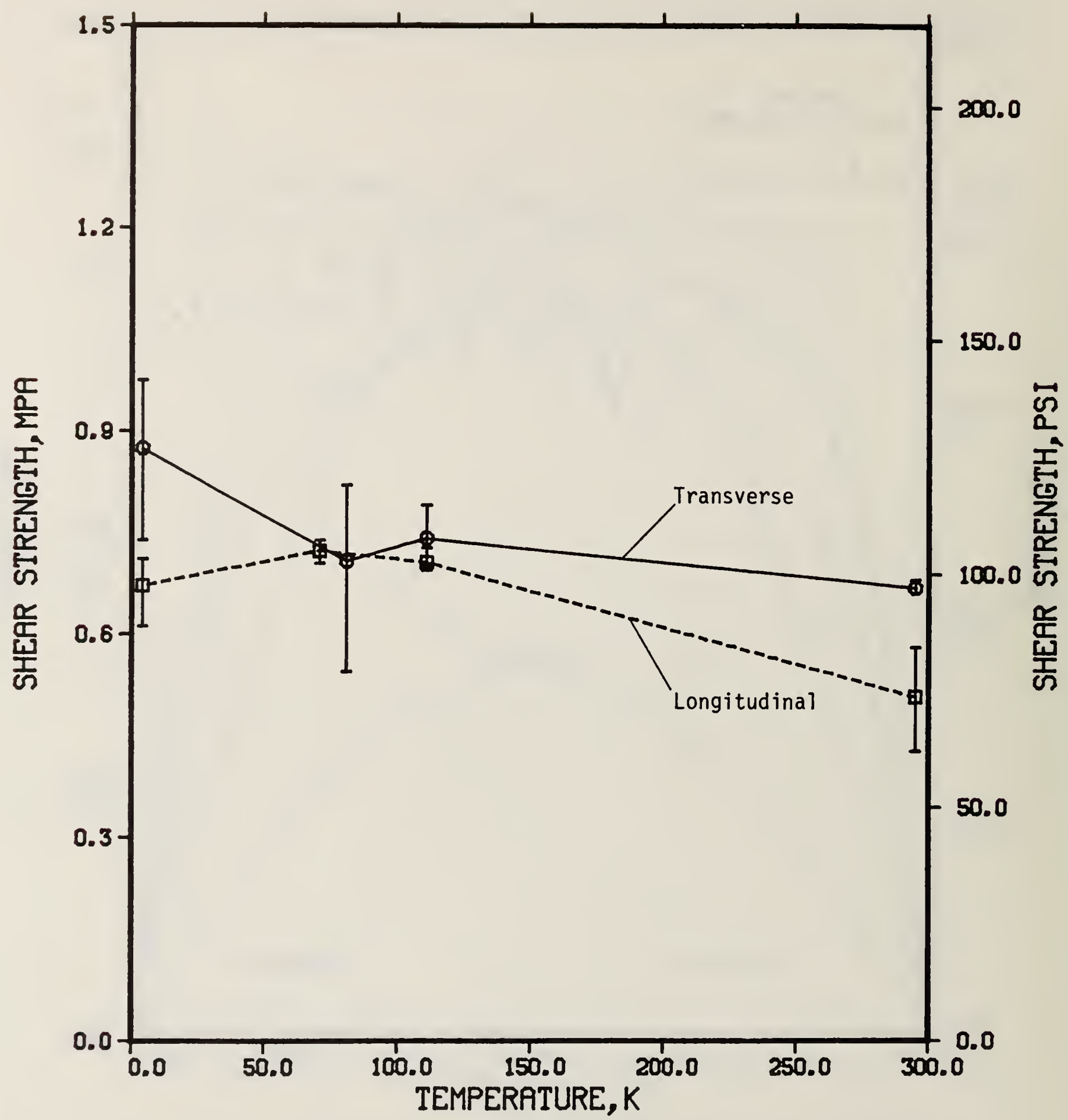

Figure 15. Shear strength versus temperature (longitudinal and transverse). 
Table 1. Tensile test results for a $64-\mathrm{kg} / \mathrm{m}^{3}$ polyurethane foam

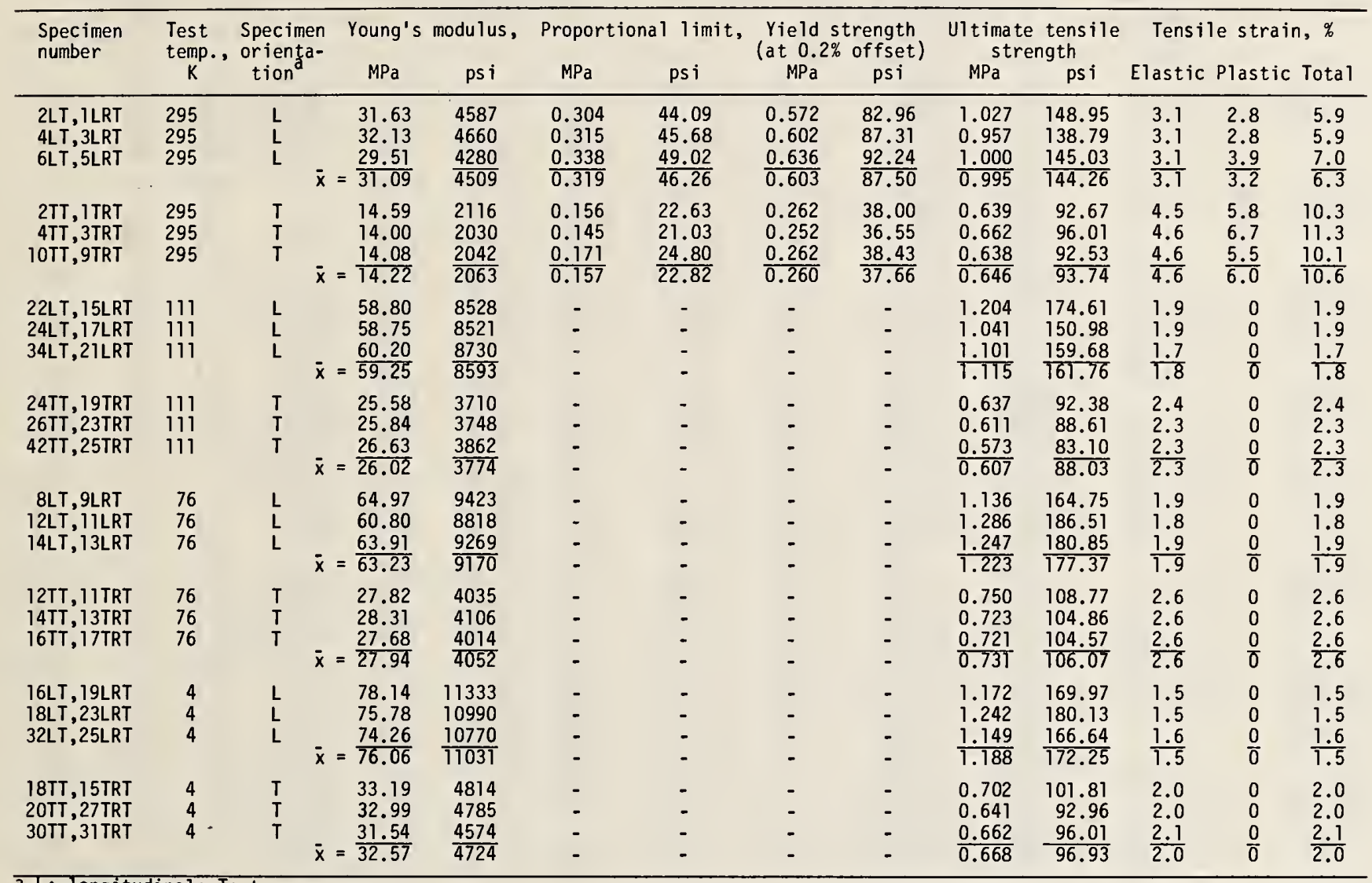


Table 2. Compressive test results for a $64-\mathrm{kg} / \mathrm{m}^{3}$ polyurethane foam

\begin{tabular}{|c|c|c|c|c|c|c|c|c|c|c|c|c|c|}
\hline $\begin{array}{l}\text { Specimen } \\
\text { number }\end{array}$ & $\begin{array}{l}\text { Test } \\
\text { temp., } \\
\text { K }\end{array}$ & $\begin{array}{l}\text { Specimen } \\
\text { orienta- } \\
\text { tion }\end{array}$ & \multicolumn{2}{|c|}{ Young's modulus, } & \multicolumn{2}{|c|}{$\begin{array}{cl}\text { Proportional limit, } \\
\mathrm{MPa}\end{array}$} & \multicolumn{2}{|c|}{$\begin{array}{l}\text { Yield strength } \\
\text { (at } 0.2 \% \text { offset), }\end{array}$} & \multicolumn{2}{|c|}{$\begin{array}{l}\text { Maximum compressiv } \\
\text { strength, } \\
\mathrm{MPa} \text { ps } \mathfrak{i}\end{array}$} & \multicolumn{3}{|c|}{$\begin{array}{l}\text { ve Compressive } \\
\text { strain, } \% \\
\text { Elastic Plastic Total }\end{array}$} \\
\hline $\begin{array}{l}26 L C(A) \\
26 L C(B) \\
28 L C(B)\end{array}$ & $\begin{array}{l}295 \\
295 \\
295\end{array}$ & $\begin{array}{l}L \\
L \\
L\end{array}$ & $\begin{array}{r}23.08 \\
20.37 \\
\quad \bar{x}=\frac{21.63}{21.69}\end{array}$ & $\begin{array}{l}3347 \\
2954 \\
3137 \\
3146\end{array}$ & $\begin{array}{l}0.355 \\
0.405 \\
0.382 \\
0.381\end{array}$ & $\begin{array}{l}51.49 \\
58.74 \\
55.40 \\
55.21\end{array}$ & $\begin{array}{l}0.498 \\
0.482 \\
0.504 \\
0.495\end{array}$ & $\begin{array}{l}72.22 \\
69.90 \\
73.10 \\
71.74\end{array}$ & $\begin{array}{l}0.668 \\
0.668 \\
0.680 \\
0.672\end{array}$ & $\begin{array}{l}96.88 \\
96.88 \\
98.62 \\
97.46\end{array}$ & $\begin{array}{l}2.8 \\
3.2 \\
3.0 \\
3.0\end{array}$ & $\begin{array}{l}2.8 \\
3.4 \\
4.4 \\
3.5\end{array}$ & $\begin{array}{l}5.6 \\
6.6 \\
7.4 \\
6.5\end{array}$ \\
\hline $\begin{array}{l}32 T C(A) \\
32 T C(B) \\
38 T C(A)\end{array}$ & $\begin{array}{l}295 \\
295 \\
295\end{array}$ & $\begin{array}{l}\mathrm{T} \\
\mathrm{T} \\
\mathrm{T}\end{array}$ & $\begin{array}{r}7.86 \\
8.30 \\
7.95 \\
\bar{x}=\quad 8.04\end{array}$ & $\begin{array}{l}1140 \\
1204 \\
1153 \\
1166\end{array}$ & $\begin{array}{l}0.121 \\
0.136 \\
0.174 \\
0.143\end{array}$ & $\begin{array}{l}17.55 \\
19.72 \\
25.24 \\
20.84\end{array}$ & $\begin{array}{l}0.222 \\
0.219 \\
0.270 \\
0.237\end{array}$ & $\begin{array}{l}32.20 \\
31.76 \\
39.16 \\
34.37\end{array}$ & $\begin{array}{r}0.360 \\
-\quad a \\
0.378 \\
0.369\end{array}$ & $\begin{array}{c}52.21 \\
- \\
54.82 \\
53.52\end{array}$ & $\begin{array}{c}4.4 \\
- \\
4.5 \\
4.5\end{array}$ & $\begin{array}{c}3.1 \\
- \\
\frac{2.6}{2.8}\end{array}$ & $\begin{array}{c}7.5 \\
-. \\
7.1 \\
7.3\end{array}$ \\
\hline $\begin{array}{l}28 L C(A) \\
28 L C(B) \\
30 L C(A)\end{array}$ & $\begin{array}{l}111 \\
111 \\
111\end{array}$ & $\begin{array}{l}L \\
L\end{array}$ & $\begin{array}{r}105.81 \\
87.19 \\
88.72 \\
\bar{x}=93.91\end{array}$ & $\begin{array}{l}15346 \\
12645 \\
12867 \\
13619\end{array}$ & $\begin{array}{l}0.715 \\
0.664 \\
0.657 \\
0.679\end{array}$ & $\begin{array}{r}103.70 \\
96.30 \\
95.28 \\
98.43\end{array}$ & $\begin{array}{l}0.817 \\
0.823 \\
0.792 \\
0.811\end{array}$ & $\begin{array}{r}118.49 \\
119.36 \\
114.86 \\
17.57\end{array}$ & $\begin{array}{l}1.078 \\
1.063 \\
1.121 \\
1.087\end{array}$ & $\begin{array}{l}156.34 \\
154.17 \\
162.58 \\
157.70\end{array}$ & $\begin{array}{l}1.0 \\
1.2 \\
1.3 \\
1.2\end{array}$ & $\begin{array}{r}8.4 \\
10.5 \\
8.3 \\
9.0\end{array}$ & $\begin{array}{r}9.4 \\
11.7 \\
9.6 \\
10.2\end{array}$ \\
\hline $\begin{array}{l}\text { 40TC }\left\{\begin{array}{l}8 \\
\mathrm{C}\end{array}\right) \\
40 \mathrm{TC} \mathrm{A}(\mathrm{A})\end{array}$ & $\begin{array}{l}111 \\
111 \\
111\end{array}$ & $\begin{array}{l}\mathrm{T} \\
\mathrm{T} \\
\mathrm{T}\end{array}$ & $\begin{array}{r}30.07 \\
30.07 \\
33.46 \\
\bar{x}= \\
31.20\end{array}$ & $\begin{array}{l}4361 \\
4361 \\
4853 \\
4525\end{array}$ & $\begin{array}{l}0.389 \\
0.362 \\
0.334 \\
0.362\end{array}$ & $\begin{array}{l}56.42 \\
52.50 \\
48.44 \\
52.45\end{array}$ & $\begin{array}{l}0.482 \\
0.409 \\
0.378 \\
0.423\end{array}$ & $\begin{array}{l}69.90 \\
59.32 \\
54.82 \\
61.35\end{array}$ & $\begin{array}{l}0.734 \\
0.722 \\
0.730 \\
0.729\end{array}$ & $\begin{array}{l}106.45 \\
104.71 \\
105.87 \\
105.68\end{array}$ & $\begin{array}{l}2.4 \\
2.3 \\
2.1 \\
2.3\end{array}$ & $\begin{array}{r}9.9 \\
10.7 \\
8.0 \\
9.5\end{array}$ & $\begin{array}{l}12.3 \\
13.0 \\
10.1 \\
11.8\end{array}$ \\
\hline $\begin{array}{l}31 L C \text { (A) } \\
31 L C \text { (8) } \\
31 L C(C)\end{array}$ & $\begin{array}{l}76 \\
76 \\
76\end{array}$ & $\begin{array}{l}L \\
L \\
L\end{array}$ & $\begin{array}{r}85.13 \\
87.77 \\
82.03 \\
\bar{x}=84.98\end{array}$ & $\begin{array}{l}12346 \\
12729 \\
11897 \\
12324\end{array}$ & $\begin{array}{l}0.846 \\
0.873 \\
0.753 \\
0.824\end{array}$ & $\begin{array}{l}122.70 \\
126.61 \\
109.21 \\
119.51\end{array}$ & $\begin{array}{l}0.970 \\
0.962 \\
0.897 \\
0.943\end{array}$ & $\begin{array}{l}140.68 \\
139.52 \\
130.09 \\
136.76\end{array}$ & $\begin{array}{l}1.179 \\
1.160 \\
1.131 \\
1.157\end{array}$ & $\begin{array}{l}170.99 \\
168.23 \\
164.03 \\
167.75\end{array}$ & $\begin{array}{l}1.4 \\
1.3 \\
1.4 \\
1.4\end{array}$ & $\begin{array}{l}7.7 \\
8.2 \\
7.2 \\
7.7\end{array}$ & $\begin{array}{l}9.1 \\
9.5 \\
\frac{8.6}{9.1}\end{array}$ \\
\hline $\begin{array}{l}38 T C(A) \\
38 T C(8) \\
49 T C(A)\end{array}$ & $\begin{array}{l}76 \\
76 \\
76\end{array}$ & $\begin{array}{l}\mathrm{T} \\
\mathrm{T} \\
\mathrm{T}\end{array}$ & $\begin{array}{r}16.80 \\
17.72 \\
16.85 \\
x=\frac{17.12}{17}\end{array}$ & $\begin{array}{l}2436 \\
2570 \\
2444 \\
2483\end{array}$ & $\begin{array}{l}0.376 \\
0.341 \\
0.349 \\
0.355\end{array}$ & $\begin{array}{l}54.53 \\
49.46 \\
50.62 \\
51.54\end{array}$ & $\begin{array}{l}0.552 \\
0.537 \\
0.539 \\
0.543\end{array}$ & $\begin{array}{l}80.06 \\
77.88 \\
78.17 \\
78.70\end{array}$ & $\begin{array}{l}0.792 \\
0.792 \\
0.781 \\
0.788\end{array}$ & $\begin{array}{l}114.86 \\
114.86 \\
113.27 \\
114.33\end{array}$ & $\begin{array}{l}4.6 \\
4.4 \\
4.5 \\
4.5\end{array}$ & $\begin{array}{l}5.9 \\
7.6 \\
7.3 \\
6.9\end{array}$ & $\begin{array}{l}10.5 \\
12.0 \\
11.8 \\
11.4\end{array}$ \\
\hline $\begin{array}{l}33 L C(A) \\
33 L C(8) \\
33 L C(C) \\
35 L C(A)\end{array}$ & $\begin{array}{l}4 \\
4 \\
4 \\
4\end{array}$ & $\begin{array}{l}L \\
L \\
L\end{array}$ & $\begin{array}{r}79.55 \\
53.17 \\
99.43 \\
\bar{x}=137.69 \\
92.46\end{array}$ & $\begin{array}{r}11537 \\
7711 \\
14420 \\
19969 \\
13409\end{array}$ & $\begin{array}{l}0.765 \\
0.850 \\
0.846 \\
- \\
0.820\end{array}$ & $\begin{array}{c}110.95 \\
123.28 \\
122.70 \\
-. \\
118.98\end{array}$ & $\begin{array}{l}0.897 \\
0.955 \\
0.947 \\
0.765 \\
0.891\end{array}$ & $\begin{array}{l}130.09 \\
138.50 \\
137.34 \\
110.95 \\
129.22\end{array}$ & $\begin{array}{l}1.156 \\
1.183 \\
1.210 \\
1.206 \\
1.189\end{array}$ & $\begin{array}{l}167.65 \\
171.57 \\
175.49 \\
174.91 \\
172.41\end{array}$ & $\begin{array}{l}1.5 \\
2.2 \\
1.2 \\
0.9 \\
1.5\end{array}$ & $\begin{array}{l}5.9 \\
7.4 \\
8.7 \\
8.5 \\
7.6\end{array}$ & $\begin{array}{l}7.4 \\
9.6 \\
9.9 \\
9.4 \\
\frac{9.1}{9}\end{array}$ \\
\hline $\begin{array}{l}35 \mathrm{TC}(8) \\
48 \mathrm{TC}(\mathrm{A}) \\
49 \mathrm{TC}(8)\end{array}$ & $\begin{array}{l}4 \\
4 \\
4\end{array}$ & $\begin{array}{l}\mathrm{T} \\
\mathrm{T} \\
\mathrm{T}\end{array}$ & $\begin{array}{r}43.48 \\
46.29 \\
45.17 \\
\bar{x}= \\
44.98\end{array}$ & $\begin{array}{l}6306 \\
6713 \\
6551 \\
6523\end{array}$ & $\begin{array}{l}0.294 \\
0.475 \\
- \\
0.384\end{array}$ & $\begin{array}{c}42.64 \\
68.89 \\
-\quad \\
55.77\end{array}$ & $\begin{array}{l}0.387 \\
0.579 \\
-\quad \\
0.483\end{array}$ & $\begin{array}{c}56.13 \\
83.97 \\
-\quad \\
70.05\end{array}$ & $\begin{array}{l}0.807 \\
0.850 \\
0.838 \\
0.832\end{array}$ & $\begin{array}{l}117.04 \\
123.28 \\
121.53 \\
120.62\end{array}$ & $\begin{array}{l}2.1 \\
2.0 \\
\frac{-}{2.0}\end{array}$ & $\begin{array}{r}8.4 \\
14.3 \\
- \\
11.4\end{array}$ & $\begin{array}{l}10.5 \\
16.3 \\
\frac{-}{13.4}\end{array}$ \\
\hline
\end{tabular}

a stress continued to increase as strain increased - no maximum reached. 
Table 3. Shear strength test results for a $64-\mathrm{kg} / \mathrm{m}^{3}$ polyurethane foam

\begin{tabular}{|c|c|c|c|c|}
\hline \multirow{2}{*}{$\begin{array}{l}\text { Specimen } \\
\text { number }\end{array}$} & \multirow{2}{*}{$\begin{array}{c}\text { Test } \\
\text { temperature, } \\
K\end{array}$} & \multirow{2}{*}{$\begin{array}{c}\text { Specimen } \\
\text { orientation }\end{array}$} & \multicolumn{2}{|c|}{ Shear strength, } \\
\hline & & & $\mathrm{MPa}$ & psi \\
\hline $\begin{array}{l}\text { SL } 4 \\
\text { SL6 } \\
\text { SL } 7\end{array}$ & $\begin{array}{l}295 \\
295 \\
295\end{array}$ & $\begin{array}{l}\mathrm{L} \\
\mathrm{L} \\
\mathrm{L}\end{array}$ & $\begin{array}{r}0.428 \\
0.581 \\
\bar{x}= \\
0.514 \\
0.508\end{array}$ & $\begin{array}{l}61.99 \\
84.19 \\
74.53 \\
73.57\end{array}$ \\
\hline $\begin{array}{l}\text { ST6 } \\
\text { ST7 } \\
\text { ST8 }\end{array}$ & $\begin{array}{l}295 \\
295 \\
295\end{array}$ & $\begin{array}{l}\mathrm{T} \\
\mathrm{T} \\
\mathrm{T}\end{array}$ & $\begin{array}{r}0.681 \\
0.661 \\
\bar{x}=\frac{0.663}{0.668}\end{array}$ & $\begin{array}{l}98.68 \\
95.90 \\
96.19 \\
96.92\end{array}$ \\
\hline $\begin{array}{l}\text { SL11 } \\
\text { SL12 } \\
\text { SL13 }\end{array}$ & $\begin{array}{l}111 \\
111 \\
111\end{array}$ & $\begin{array}{l}\mathrm{L} \\
\mathrm{L} \\
\mathrm{L}\end{array}$ & $\begin{array}{r}0.697 \\
0.728 \\
\bar{x}=\frac{0.694}{0.706}\end{array}$ & $\begin{array}{l}101.01 \\
105.54 \\
100.67 \\
102.41\end{array}$ \\
\hline $\begin{array}{l}\text { ST11 } \\
\text { ST12 } \\
\text { ST13 }\end{array}$ & $\begin{array}{l}111 \\
111 \\
111\end{array}$ & $\begin{array}{l}\mathrm{T} \\
\mathrm{T} \\
\mathrm{T}\end{array}$ & $\begin{array}{r}0.704 \\
0.729 \\
0.791 \\
\bar{x}= \\
0.741\end{array}$ & $\begin{array}{l}102.09 \\
105.65 \\
114.67 \\
107.47\end{array}$ \\
\hline $\begin{array}{l}\text { SL1 } \\
\text { SL2 } \\
\text { SL3 }\end{array}$ & $\begin{array}{l}76 \\
76 \\
76\end{array}$ & $\begin{array}{l}\mathrm{L} \\
\mathrm{L} \\
\mathrm{L}\end{array}$ & $\begin{array}{l}0.727 \\
0.739 \\
\bar{x}= \\
0.705 \\
0.724\end{array}$ & $\begin{array}{l}105.38 \\
107.13 \\
102.23 \\
104.91\end{array}$ \\
\hline $\begin{array}{l}\text { ST4 } \\
\text { ST9 } \\
\text { ST10 }\end{array}$ & $\begin{array}{l}76 \\
76 \\
76\end{array}$ & $\begin{array}{l}T \\
T \\
T\end{array}$ & $\begin{array}{r}0.821 \\
0.546 \\
0.759 \\
\bar{x}=\frac{0.709}{0}\end{array}$ & $\begin{array}{r}119.07 \\
79.19 \\
110.00 \\
102.75\end{array}$ \\
\hline $\begin{array}{l}\text { SL8 } \\
\text { SL9 } \\
\text { SL } 10\end{array}$ & $\begin{array}{l}4 \\
4 \\
4\end{array}$ & $\begin{array}{l}\mathrm{L} \\
\mathrm{L} \\
\mathrm{L}\end{array}$ & $\begin{array}{r}0.612 \\
0.691 \\
0.712 \\
0.672\end{array}$ & $\begin{array}{r}88.70 \\
100.18 \\
103.20 \\
97.36\end{array}$ \\
\hline $\begin{array}{l}\text { ST1 } \\
\text { ST3 } \\
\text { ST5 }\end{array}$ & $\begin{array}{l}4 \\
4 \\
4\end{array}$ & $\begin{array}{l}T \\
T \\
T\end{array}$ & $\begin{array}{r}0.909 \\
0.739 \\
0.975 \\
\bar{x}=\frac{0.874}{}\end{array}$ & $\begin{array}{l}131.78 \\
107.08 \\
141.37 \\
126.74\end{array}$ \\
\hline
\end{tabular}


Table 4. Sumnary of tensile test results for a $64-\mathrm{kg} / \mathrm{m}^{3}$ polyurethane foam (average values)

\begin{tabular}{|c|c|c|c|c|c|}
\hline $\begin{array}{l}\text { Material } \\
\text { property }\end{array}$ & $\begin{array}{c}\text { Specimen } \\
\text { orientation }\end{array}$ & $295 \mathrm{~K}$ & $\begin{array}{l}\text { Temperature } \\
111 \mathrm{k}\end{array}$ & $76 \mathrm{~K}$ & $4 \mathrm{~K}$ \\
\hline $\begin{array}{l}\text { Young's } \\
\text { modulus, } \\
\mathrm{MPa} \text { (psi) }\end{array}$ & $\stackrel{\mathrm{L}}{\mathrm{T}}$ & $\begin{array}{l}31.09(4,510) \\
14.22(2,060)\end{array}$ & $\begin{array}{ll}59.25 & (8,590) \\
26.02 & (3,770)\end{array}$ & $\begin{array}{l}63.23(9,170) \\
27.94(4,050)\end{array}$ & $\begin{array}{l}76.06(11,030) \\
32.57(4,720)\end{array}$ \\
\hline $\begin{array}{l}\text { Proportional } \\
\text { limit, } \\
\text { MPa (psi) }\end{array}$ & $\stackrel{\mathrm{L}}{\mathrm{T}}$ & $\begin{array}{l}0.319(46.26) \\
0.157(22.82)\end{array}$ & - & - & - \\
\hline $\begin{array}{l}\text { Yield strength } \\
(0.2 \% \text { offset), } \\
\mathrm{MPa} \text { (psi) }\end{array}$ & $\stackrel{\mathrm{L}}{\mathrm{T}}$ & $\begin{array}{l}0.603(87.50) \\
0.260(37.66)\end{array}$ & - & 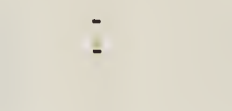 & - \\
\hline $\begin{array}{l}\text { Ultimate ten- } \\
\text { sile strength, } \\
\mathrm{MPa} \text { (psi) }\end{array}$ & $\stackrel{L}{T}$ & $\begin{array}{l}0.995(144.26) \\
0.646(93.74)\end{array}$ & $\begin{array}{l}1.115(161.76) \\
0.607(88.03)\end{array}$ & $\begin{array}{l}1.223(177.37) \\
0.731(106.07)\end{array}$ & $\begin{array}{l}1.188(172.25) \\
0.668(96.93)\end{array}$ \\
\hline $\begin{array}{l}\text { Tensile strain, \% } \\
\text { Elastic } \\
\text { Plastic } \\
\text { Total }\end{array}$ & $\mathrm{L}$ & $\begin{array}{l}3.1 \\
3.2 \\
6.3\end{array}$ & $\begin{array}{r}1.8 \\
0 \\
1.8\end{array}$ & $\begin{array}{r}1.9 \\
0 \\
1.9\end{array}$ & $\begin{array}{r}1.5 \\
0 \\
1.5\end{array}$ \\
\hline $\begin{array}{l}\text { Elastic } \\
\text { Plastic } \\
\text { Total }\end{array}$ & $T$ & $\begin{array}{r}4.6 \\
6.0 \\
10.6\end{array}$ & $\begin{array}{r}2.3 \\
0 \\
2.3\end{array}$ & $\begin{array}{r}2.6 \\
0 \\
2.6\end{array}$ & $\begin{array}{r}2.0 \\
0 \\
2.0\end{array}$ \\
\hline
\end{tabular}

Table 5. Summary of compressive test results for a $64-\mathrm{kg} / \mathrm{m}^{3}$ polyurethane foam (average values)

\begin{tabular}{|c|c|c|c|c|c|}
\hline $\begin{array}{l}\text { Material } \\
\text { property }\end{array}$ & $\begin{array}{c}\text { Specimen } \\
\text { orientation }\end{array}$ & $295 \mathrm{~K}$ & $111 k^{\text {Tempe }}$ & $76 \mathrm{~K}$ & $4 \mathrm{~K}$ \\
\hline $\begin{array}{l}\text { Young's } \\
\text { modulus, } \\
\text { MPa (psi) }\end{array}$ & $\stackrel{L}{T}$ & $\begin{array}{r}21.69(3,150) \\
8.04(1,170)\end{array}$ & $\begin{array}{r}93.91(13,620) \\
31.20(4,530)\end{array}$ & 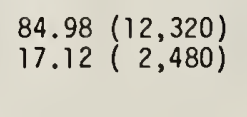 & 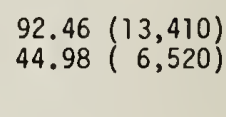 \\
\hline $\begin{array}{l}\text { Proportional } \\
\text { limit, } \\
\text { MPa (psi) }\end{array}$ & $\stackrel{L}{T}$ & $\begin{array}{ll}0.381 & (55.21) \\
0.143 & (20.84)\end{array}$ & $\begin{array}{l}0.679(98.43) \\
0.362(52.45)\end{array}$ & $\begin{array}{l}0.824(119.51) \\
0.355(51.54)\end{array}$ & $\begin{array}{l}0.820(118.98) \\
0.384(55.77)\end{array}$ \\
\hline 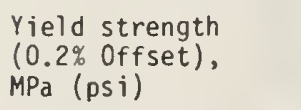 & $\stackrel{L}{T}$ & $\begin{array}{l}0.495(71.74) \\
0.237(34.37)\end{array}$ & $\begin{array}{l}0.811(117.57) \\
0.423(61.35)\end{array}$ & $\begin{array}{l}0.943(136.76) \\
0.543(78.70)\end{array}$ & $\begin{array}{l}0.891(129.22) \\
0.483(70.05)\end{array}$ \\
\hline $\begin{array}{l}\text { Maximum compres- } \\
\text { sive strength, } \\
M P a(p s i)\end{array}$ & $\stackrel{L}{T}$ & $\begin{array}{l}0.672(97.46) \\
0.369(53.52)\end{array}$ & $\begin{array}{l}1.087(157.70) \\
0.729(105.68)\end{array}$ & $\begin{array}{l}1.157(167.75) \\
0.788(114.33)\end{array}$ & $\begin{array}{l}1.189(172.41) \\
0.832(120.62)\end{array}$ \\
\hline $\begin{array}{l}\text { Compressive strain, } \\
\text { Elastic } \\
\text { Plastic } \\
\text { Total }\end{array}$ & $\%$ & $\begin{array}{l}3.0 \\
3.5 \\
6.5\end{array}$ & $\begin{array}{r}1.2 \\
9.0 \\
10.2\end{array}$ & $\begin{array}{l}1.4 \\
7.7 \\
9.1\end{array}$ & $\begin{array}{l}1.5 \\
7.6 \\
9.1\end{array}$ \\
\hline $\begin{array}{l}\text { Elastic } \\
\text { Plastic } \\
\text { Total }\end{array}$ & $T$ & $\begin{array}{l}4.5 \\
2.8 \\
7.3\end{array}$ & $\begin{array}{r}2.3 \\
9.5 \\
11.8\end{array}$ & $\begin{array}{r}4.5 \\
6.9 \\
11.4\end{array}$ & $\begin{array}{r}2.0 \\
11.4 \\
13.4\end{array}$ \\
\hline
\end{tabular}


Table 6. Summary of shear strength test results for a $64-\mathrm{kg} / \mathrm{m}^{3}$ polyurethane foam (average values)

\begin{tabular}{cccc}
\hline $\begin{array}{c}\text { Test } \\
\text { temperature, K }\end{array}$ & Specimen orientation & \multicolumn{2}{c}{ Shear strength, } \\
$\mathrm{MPa}$ & 0.508 & 73.57 \\
\hline \multirow{2}{*}{295} & $\mathrm{~L}$ & 0.668 & 96.92 \\
& $\mathrm{~T}$ & 0.706 & 102.41 \\
111 & $\mathrm{~L}$ & 0.741 & 107.47 \\
& $\mathrm{~T}$ & 0.724 & 104.91 \\
76 & $\mathrm{~L}$ & 0.709 & 102.75 \\
& $\mathrm{~T}$ & 0.672 & 97.36 \\
4 & $\mathrm{~L}$ & 0.874 & 126.74 \\
\hline
\end{tabular}


NBS.114A IREV. 2-8C)

\begin{tabular}{|c|c|}
\hline U.S. DEPT. OF COMM. & 1. PUBLICATION OR \\
BIBLIOGRAPHIC DATA & REPORT NO. \\
SHEET (See instructions) & NBSIR 83-1684 \\
\hline
\end{tabular}

4. TITLE AND SUBTITLE

Tensile, Compressive, and Shear Properties of a $64-\mathrm{kg} / \mathrm{m}^{3}$ Polyurethane Foam at Low Temperatures

5. $A \cup T H O R(S)$
6. PERFORMING ORGANIZATION (If joint or other than NBS, see instructions)

NATIONAL BUREAU OF STANDARDS

DEPARTMENT OF COMMERCE

WASHINGTON, D.C. 20234

9. SPONSORING ORGANIZATION NAME AND COMPLETE ADDRESS (Street, City, State, ZIP)

Gas Research Institute

8600 West Bryn Mawr Ave.

Chicago, IL 60631

10. SUPPLEMENTARY NOTES

D Document describes a computer program; SF-185, FIPS Software Summary, is attached.

11. ABSTRACT (A 200-word or less factual summary of most significant information. If document includes a significant bibliography or literature survey. mention it here)

Polyurethane foam, having a density of $64-\mathrm{kg} / \mathrm{m}^{3}$, was tested at $295,111,76$, and $4 \mathrm{~K}$ The material properties reported are Young's modulus, proportional limit, yield strength (at $0.2 \%$ offset), tensile, shear, and compressive strengths, and elongation (elastic and plastic). To perform these tests, a unique apparatus was developed. This apparatus permits tension, compression, and shear testing of materials at any temperatufe ranging from 295 to $1.8 \mathrm{~K}$. Strain is measured with a concentric, overlapping-cylinder capacitance extensometer that is highly sensitive and linear in output.

12. KEY WORDS (Six to twelve entries: alphabetical order: capitalize only proper names; and separate key words by semicolons)

compressive strength; elongation; foam; insulation; low temperatures; mechanical properties; proportional litiit; shear strength; tensile strength; yield strength; 13. AVATLÁBILITY motus.

[x] Unlimited

For Official Distribution. Do Not Release to NTIS

Order From Superintendent of Documents, U.S. Government Printing Office, Washington, D.C. 20402.

[X] Order From National Technical Information Service (NTIS), Springfield, VA. 22161

14. NO. OF PRINTED PAGES

33

15. Price

$\$ 8.50$ 



